\title{
RIGHT LIMITS AND REFLECTIONLESS MEASURES FOR CMV MATRICES
}

\author{
JONATHAN BREUER, ERIC RYCKMAN, AND MAXIM ZINCHENKO
}

\begin{abstract}
We study CMV matrices by focusing on their right-limit sets. We prove a CMV version of a recent result of Remling dealing with the implications of the existence of absolutely continuous spectrum, and we study some of its consequences. We further demonstrate the usefulness of right limits in the study of weak asymptotic convergence of spectral measures and ratio asymptotics for orthogonal polynomials by extending and refining earlier results of Khrushchev. To demonstrate the analogy with the Jacobi case, we recover corresponding previous results of Simon using the same approach.
\end{abstract}

\section{INTRODUCTION}

This paper considers some issues in the spectral theory of CMV matrices viewed through the lens of the notion of right limits. In particular, a central theme will be the fact that one may use the properties of right limits of a given CMV matrix to deduce relations between the asymptotics of its entries and its spectral measure.

CMV matrices (see Definition 1.2 below) were named after Cantero, Moral and Velázquez [4] and may be described as the unitary analog of Jacobi matrices: they arise naturally in the theory of orthogonal polynomials on the unit circle (OPUC) in much the same way that Jacobi matrices arise in the theory of orthogonal polynomials on the real line (OPRL).

Two related topics will be at the focus of our discussion. The first is the extension to the CMV setting of a collection of results, proven recently by Remling [33], describing various consequences of the existence of absolutely continuous spectrum of Jacobi matrices. The second topic is the simplification of various elements of Khrushchev's theory of weak limits of spectral measures, through the understanding that the matrices at the center of attention have right limits in a very special class.

As we shall see, these two subjects are intimately connected through the notion of reflectionless whole-line CMV matrices. This is a concept that has been extensively investigated in recent years, in the context of both CMV and Jacobi matrices ([6], [7], [9]-[11], [14], [17]-[22], [24]-[29], [32], [33], [38]-[42]) and was seen to have numerous applications in their spectral theory. There are various definitions of this notion, all of which turn out to be equivalent in the Jacobi matrix case. We shall show that this is not true in the CMV case. In particular, we construct an example of a whole-line CMV matrix that is not reflectionless in the spectral-theoretic sense, while all of its diagonal spectral measures are reflectionless in the measure-theoretic sense. We will show, however, that this may only happen for a very limited class

Date: June 1, 2018.

2000 Mathematics Subject Classification. 42C05, 30E10, 34L40.

Key words and phrases. Right limits, reflectionless property, CMV operators, ratio asymptotics. 
of CMV matrices. Their existence in the CMV case, together with Remling's Theorem (Theorem 1.4 below), provides for a simple proof of Khrushchev's Theorem (Theorem 1.9 below).

We should remark that ours is not the first paper to deal with right limits of CMV matrices. For other examples and related results, see for instance [15] and [23].

In order to describe our results, some notation is needed: given a probability measure, $\mu$, on the boundary of the unit disc, $\partial \mathbb{D}$, we let $\left\{\Phi_{n}(z)\right\}_{n=0}^{\infty}$ and $\left\{\varphi_{n}(z)\right\}_{n=0}^{\infty}$ denote the monic orthogonal and the orthonormal polynomials one gets by applying the Gram-Schmidt procedure to $1, z, z^{2}, \ldots$ (we assume throughout that the support of $\mu$ is an infinite set so the polynomial sequences are indeed infinite). The $\Phi_{n}$ satisfy the Szegö recurrence equation:

$$
\Phi_{n+1}(z)=z \Phi_{n}(z)-\overline{\alpha_{n}} \Phi_{n}^{*}(z)
$$

where $\left\{\alpha_{n}\right\}_{n=0}^{\infty}$ is a sequence of parameters satisfying $\left|\alpha_{n}\right|<1$ and $\Phi_{n}^{*}(z)=$ $z^{n} \overline{\Phi_{n}(1 / \bar{z})}$. We call $\left\{\alpha_{n}\right\}_{n=0}^{\infty}$ the Verblunsky coefficients associated with $\mu$. As is well known [36], the sequence $\left\{\alpha_{n}\right\}_{n=0}^{\infty}$ may be used to construct a semi-infinite 5-diagonal matrix, $\mathcal{C}$, (called the CMV matrix) such that the operator of multiplication by $z$ on $L^{2}(\partial \mathbb{D}, d \mu)$ is unitarily equivalent to the operator $\mathcal{C}$ on $\ell^{2}\left(\mathbb{Z}_{+}\right)$ $\left(\mathbb{Z}_{+}=\{0,1,2, \ldots\}\right)$. Explicitly, $\mathcal{C}$ is given by

$$
\mathcal{C}=\left(\begin{array}{cccccc}
\bar{\alpha}_{0} & \bar{\alpha}_{1} \rho_{0} & \rho_{1} \rho_{0} & 0 & 0 & \ldots \\
\rho_{0} & -\bar{\alpha}_{1} \alpha_{0} & -\rho_{1} \alpha_{0} & 0 & 0 & \ldots \\
0 & \bar{\alpha}_{2} \rho_{1} & -\bar{\alpha}_{2} \alpha_{1} & \bar{\alpha}_{3} \rho_{2} & \rho_{3} \rho_{2} & \ldots \\
0 & \rho_{2} \rho_{1} & -\rho_{2} \alpha_{1} & -\bar{\alpha}_{3} \alpha_{2} & -\rho_{3} \alpha_{2} & \ldots \\
0 & 0 & 0 & \bar{\alpha}_{4} \rho_{3} & -\bar{\alpha}_{4} \alpha_{3} & \ldots \\
\ldots & \cdots & \ldots & \ldots & \ldots & \ldots
\end{array}\right)
$$

with $\rho_{n}=\left(1-\left|\alpha_{n}\right|^{2}\right)^{1 / 2}$.

Now, for a probability measure $\mu$ on $\partial \mathbb{D}$, let $d \mu(\theta)=w(\theta) d \theta+d \mu_{\operatorname{sing}}(\theta)$ be the decomposition into its absolutely continuous and singular parts (with respect to the Lebesgue measure). If $\mathcal{C}$ is the corresponding CMV matrix, we define the essential support of the absolutely continuous spectrum of $\mathcal{C}$ to be the set $\Sigma_{\mathrm{ac}}(\mathcal{C}) \equiv$ $\{\theta \mid w(\theta)>0\}$. Clearly, $\Sigma_{\text {ac }}(\mathcal{C})$ is only defined up to sets of Lebesgue measure zero, so the symbol and the name should be understood as representing elements in an equivalence class of sets rather than a particular set. For the sake of simplicity we will ignore this point in our discussion.

The first part of this paper deals with proving the analog of Remling's Theorem (Theorem 1.4 in [33]) for CMV matrices and deriving some consequences. In a nutshell, Remling's Theorem for CMV matrices says that for any given CMV matrix, $\mathcal{C}$, all of its right limits are reflectionless on $\Sigma_{\mathrm{ac}}(\mathcal{C})$ (see Definitions 1.2 and 1.3 and Theorem 1.4 below). Here is a consequence that will also provide the link to Khrushchev's theory (the Jacobi analog was stated and proved in [33]):

Theorem 1.1. Let $\left\{\alpha_{n}\right\}_{n=0}^{\infty}$ and $\left\{\widetilde{\alpha}_{n}\right\}_{n=0}^{\infty}$ be two sequences of Verblunsky coefficients such that (with $\Delta_{n}=\widetilde{\alpha}_{n}-\alpha_{n}$ )

(i) $\left|\alpha_{n}\right|<1,\left|\widetilde{\alpha}_{n}\right|<1$ for all $n$.

(ii) There exist sequences $\left\{m_{j}\right\}_{j=1}^{\infty},\left\{n_{j}\right\}_{j=1}^{\infty}$ with $n_{j}-m_{j} \rightarrow \infty$ so that

$$
\lim _{j \rightarrow \infty} \sup _{m_{j} \leq n<n_{j}}\left|\Delta_{n}\right|=0 .
$$


(iii) $\lim \sup _{j \rightarrow \infty}\left|\Delta_{n_{j}}\right|>0$.

Furthermore, let $\mathcal{C}$ and $\widetilde{\mathcal{C}}$ denote the $C M V$ matrices of $\left\{\alpha_{n}\right\}_{n=0}^{\infty}$ and $\left\{\widetilde{\alpha}_{n}\right\}_{n=0}^{\infty}$ respectively. Then

$$
\operatorname{Leb}\left(\Sigma_{\mathrm{ac}}(\mathcal{C}) \cap \Sigma_{\mathrm{ac}}(\widetilde{\mathcal{C}})\right)=0
$$

where Leb $(\cdot)$ denotes Lebesgue measure. In particular, if $\mathcal{C}$ is associated with a sequence of Verblunsky coefficients satisfying

$$
\forall k \geq 1 \quad \lim _{n \rightarrow \infty} \alpha_{n} \alpha_{n+k}=0, \quad \limsup _{n \rightarrow \infty}\left|\alpha_{n}\right|>0,
$$

then $\mathcal{C}$ has purely singular spectrum.

In order to state Remling's Theorem we need some more terminology.

Definition 1.2. Given a sequence of Verblunsky coefficients $\left\{\alpha_{n}\right\}_{n=0}^{\infty}$, a doublyinfinite sequence of parameters $\left\{\widetilde{\alpha}_{n}\right\}_{n \in \mathbb{Z}}$ with $\left|\widetilde{\alpha}_{n}\right| \leq 1$ is called a right limit of $\left\{\alpha_{n}\right\}_{n=0}^{\infty}$ if there is a sequence of integers $n_{j} \rightarrow \infty$ such that $\forall n \in \mathbb{Z}, \widetilde{\alpha}_{n}=$ $\lim _{j \rightarrow \infty} \alpha_{n+n_{j}}$.

Since a sequence of Verblunsky coefficients is always bounded, by compactness it always has at least one (and perhaps many) right limits. Given a doubly infinite sequence $\left\{\widetilde{\alpha}_{n}\right\}_{n \in \mathbb{Z}}$, one may also define a corresponding unitary matrix on $\ell^{2}(\mathbb{Z})$, extending the half-line matrices to the left and top (see (3.1) for the precise form). We call such a matrix the corresponding whole-line CMV matrix and denote it by $\mathcal{E}$. For this reason, we shall often refer to a doubly infinite sequence of numbers $\left\{\widetilde{\alpha}_{n}\right\}_{n \in \mathbb{Z}}$ with $\left|\widetilde{\alpha}_{n}\right| \leq 1$ as a (doubly infinite) sequence of Verblunsky coefficients. If $\left\{\widetilde{\alpha}_{n}\right\}_{n \in \mathbb{Z}}$ is a right limit of $\left\{\alpha_{n}\right\}_{n=0}^{\infty}$, we refer to the corresponding whole-line CMV matrix as a right limit of the half-line CMV matrix associated with $\left\{\alpha_{n}\right\}_{n=0}^{\infty}$.

Recall that any probability measure $\mu$ on $\partial \mathbb{D}$ may be naturally associated with a Schur function $f$ (an analytic function on $\mathbb{D}$ satisfying $\sup _{z \in \mathbb{D}}|f(z)| \leq 1$ ) and a Carathéodory function $F$ (an analytic function on $\mathbb{D}$ satisfying $F(0)=1$ and $\operatorname{Re} F(z)>0$ on $\mathbb{D})$. This is given by

$$
\frac{1+z f(z)}{1-z f(z)}=F(z)=\int_{0}^{2 \pi} \frac{e^{i \theta}+z}{e^{i \theta}-z} d \mu(\theta) .
$$

The correspondence is $1-1$ and onto. By a classical result, $\lim _{r \uparrow 1} F\left(r e^{i \theta}\right)$ and $\lim _{r \uparrow 1} f\left(r e^{i \theta}\right)$ exist Lebesgue a.e. on $\partial \mathbb{D}$. We denote them by $F\left(e^{i \theta}\right)$ and $f\left(e^{i \theta}\right)$ respectively and, when there is no danger of confusion, simply by $F(z)$ or $f(z)$ for $z \in \partial \mathbb{D}$.

Given a Schur function, $f$, let $f_{0}=f$ and define a sequence of Schur functions $f_{n}$ and parameters $\gamma_{n} \in \mathbb{D}$ by

$$
z f_{n+1}(z)=\frac{f_{n}(z)-\gamma_{n}}{1-\bar{\gamma}_{n} f_{n}(z)}, \quad \gamma_{n}=f_{n}(0) .
$$

If for some $n,\left|\gamma_{n}\right|=1$, we stop and the Schur function is a finite Blaschke product. Otherwise, we continue. It is known [36] that this process (known as the Schur algorithm) sets up a 1-1 correspondence between Schur functions $f$ and parameter sequences $\gamma_{n} \in \mathbb{D}$, so given any sequence $\gamma_{n} \in \mathbb{D}$ there is a unique Schur function $f\left(z ; \gamma_{0}, \gamma_{1}, \ldots\right)$ associated to it in the above way. The $\gamma$ 's are frequently termed the Schur parameters associated to $f$ (or equivalently, to $\mu$ or $F$ ). Geronimus's 
Theorem [8] says that $\gamma_{n}=\alpha_{n}$ (the Verblunsky coefficients of $\mu$ appearing above). Finally, note that by definition

$$
f_{n}\left(z ; \alpha_{0}, \alpha_{1}, \ldots\right)=f\left(z ; \alpha_{n}, \alpha_{n+1}, \ldots\right) .
$$

For a doubly infinite sequence of Verblunsky coefficients, $\left\{\alpha_{n}\right\}_{n \in \mathbb{Z}}$ (some of which may lie on $\partial \mathbb{D})$, we define two sequences of Schur functions:

$$
f_{+}(z, n)=f\left(z ; \alpha_{n}, \alpha_{n+1}, \ldots\right) \quad \text { and } \quad f_{-}(z, n)=f\left(z ;-\overline{\alpha_{n-1}},-\overline{\alpha_{n-2}}, \ldots\right)
$$

where as usual, if one of the $\alpha$ 's lies in $\partial \mathbb{D}$ then we stop the Schur algorithm at that point and the corresponding Schur function is a finite Blaschke product.

Definition 1.3. Let $\left\{\alpha_{n}\right\}_{n \in \mathbb{Z}}$ be a doubly-infinite sequence of Verblunsky coefficients and let $\mathcal{E}$ be the associated whole-line CMV matrix. Given a Borel set $A \subseteq \partial \mathbb{D}$, we will say $\mathcal{E}$ is reflectionless on $A$ if for all $n \in \mathbb{Z}$,

$$
z f_{+}(z, n)=\overline{f_{-}(z, n)}
$$

for Lebesgue almost every $z \in A$. By the Schur algorithm, one can easily see that "for all $n \in \mathbb{Z}$ " may be replaced with "for some $n \in \mathbb{Z}$."

Remark. The analogous definition for whole-line Jacobi matrices involves a similar relationship between the left and right $m$-functions.

The following is the CMV version of Remling's Theorem:

Theorem 1.4 (Remling's Theorem for CMV matrices). Let $\mathcal{C}$ be a half-line $C M V$ matrix, and let $\Sigma_{\mathrm{ac}}(\mathcal{C})$ be the essential support of the absolutely continuous part of the spectral measure. Then every right limit of $\mathcal{C}$ is reflectionless on $\Sigma_{\mathrm{ac}}(\mathcal{C})$.

Remling's proof in the Jacobi case relies on previous work by Breimesser and Pearson $[1,2]$ concerning convergence of boundary values for Herglotz functions. We will prove Theorem 1.4 using the analogous theory for Schur functions. For a CMV matrix, $\mathcal{C}$, recall that its essential spectrum, $\sigma_{\text {ess }}(\mathcal{C})$, is its spectrum with the isolated points removed. The following extension of a celebrated theorem of Rakhmanov is a simple corollary of Theorem 1.4:

Theorem 1.5. Assume $\Sigma_{\mathrm{ac}}(\mathcal{C})=\sigma_{\mathrm{ess}}(\mathcal{C})=A$ where $\sigma_{\mathrm{ess}}(\mathcal{C})$ is the essential spectrum of $\mathcal{C}$. Then for any right limit $\mathcal{E}$ of $\mathcal{C}, \sigma(\mathcal{E})=A$ and $\mathcal{E}$ is reflectionless on $A$.

Remark. In the case that $A$ is a finite union of intervals, the corresponding class of whole-line CMV matrices is called the isospectral torus of $A$, since it has a natural torus structure $[12,28]$. If $A=\partial \mathbb{D}$, the isospectral torus is known to consist of a single point - the CMV matrix with Verblunsky coefficients all equal to zero [12]. Thus, one gets Rakhmanov's Theorem [30, 31] as a corollary.

Proof of Theorem 1.5. Let $\mathcal{E}$ be a right limit of $\mathcal{C}$ and $\left\{\delta_{n}\right\}_{n \in \mathbb{Z}}$ be the standard orthonormal bais for $\ell^{2}(\mathbb{Z})$. For $\psi=\sum_{n \in \mathbb{Z}} 2^{-|n|} \delta_{n}$, let $d \mu_{\psi}(\theta)=w_{\psi}(\theta) d \theta+d \mu_{\psi, \text { sing }}$ be the spectral measure of $\psi$ and $\mathcal{E}$. Let $\Sigma_{\text {ac }}(\mathcal{E})=\left\{\theta \mid w_{\psi}(\theta)>0\right\}$ (defined, again, up to sets of Lebesgue measure zero).

By Theorem 1.4, $A \subseteq \Sigma_{\text {ac }}(\mathcal{E})$ (up to a set of Lebesgue measure zero), since the reflectionless condition implies positivity of the real part of the Carathéodory function associated with $d \mu_{\psi}$. Also, $\sigma(\mathcal{E}) \subseteq \sigma_{\text {ess }}(\mathcal{C})=A$ by approximate-eigenvector arguments (see for instance [23]). Since obviously $\Sigma_{\text {ac }}(\mathcal{E}) \subseteq \sigma(\mathcal{E})$, we have equality throughout. The reflectionless condition now follows from Theorem 1.4. 
Remark. Using Theorem 1.4 and a bit of work, one can also derive parts of Kotani theory for ergodic CMV matrices (see for instance [37, Sect. 10.11]). Remling also obtains deterministic versions of these results for Jacobi matrices (see [33, Thm's 1.1 and 1.2]). His proofs extend directly to the CMV case we are considering, so we will not pursue this here.

Corresponding to the notion of reflectionless operators, there is also the notion of reflectionless measures:

Definition 1.6. A probability measure $\mu$ on $\partial \mathbb{D}$ is said to be reflectionless on a Borel set $A \subseteq \partial \mathbb{D}$ if the corresponding Carathéodory function $F$ has $\operatorname{Im} F\left(e^{i \theta}\right)=0$ for Lebesgue a.e. $e^{i \theta} \in A$.

Remark. The analogous definition for measures on the real line involves the vanishing of the real part of the Borel (a.k.a. Cauchy or Stieltjes) transform of $\mu$ (see for instance [43]).

Remark. There is also a natural dynamical notion for when an operator is reflectionless. For the relationship between this and spectral theory see [3].

Reflectionless Jacobi matrices and reflectionless measures on $\mathbb{R}$ are related in the following way: given a whole-line Jacobi matrix, $H$, let $\mu_{n}$ be the spectral measure of $H$ and $\delta_{n}\left(\delta_{n} \in \ell^{2}(\mathbb{Z})\right.$ is defined by $\delta_{n}(j)=\delta_{n, j}$ with $\delta_{n, j}$ the Kronecker delta). Then $H$ is reflectionless on $A \subseteq \mathbb{R}$ if and only if $\mu_{n}$ are reflectionless on $A$ for all $n \in \mathbb{Z}$ (again, see [43]). A fact we would like to emphasize in this paper is that the analogous statement does not hold for CMV matrices.

Example 1.7. Fix $j_{0} \in \mathbb{Z}$ and some $0<|\beta|<1$, and let $\left\{\alpha_{n}\right\}_{n \in \mathbb{Z}}$ be the sequence of Verblunsky coefficients defined by

$$
\alpha_{n}= \begin{cases}\beta & n=j_{0} \\ 0 & \text { otherwise. }\end{cases}
$$

Let $\mathcal{E}$ be the CMV matrix for these $\alpha$ 's. From the Schur algorithm we see

$$
f(z ; 0,0, \ldots)=0 \text { and } f(z ; \beta, 0,0, \ldots)=\beta,
$$

so $\mathcal{E}$ is not reflectionless anywhere.

On the other hand, let $\mu_{n}$ be the spectral measure of $\mathcal{E}$ and $\delta_{n}$, and let $f(z, n)$ its corresponding Schur function. It is shown in [13] (see also [18] for the analogous formula in the half-line case) that

$$
f(z, n)=f_{+}(z, n) f_{-}(z, n), \quad z \in \mathbb{D}, n \in \mathbb{Z} .
$$

Thus, for any $n \in \mathbb{Z},(1.8)$ implies $d \mu_{n}(\theta)=\frac{d \theta}{2 \pi}$. In particular, $\mu_{n}$ is reflectionless on all of $\partial \mathbb{D}$ while $\mathcal{E}$ is not reflectionless on any subset of positive Lebesgue measure.

We will show, however, that this is the only example of such behavior:

Theorem 1.8. Let $\mathcal{E}$ be the whole-line $C M V$ matrix corresponding to the sequence $\left\{\alpha_{n}\right\}_{n \in \mathbb{Z}}$, satisfying $\alpha_{n} \neq 0$ for at least two different $n \in \mathbb{Z}$. Then $\mathcal{E}$ is reflectionless on $A \subseteq \partial \mathbb{D}$ if and only if $\mu_{n}$ is reflectionless on $A$ for all $n$.

The connection between the above result and Khrushchev's theory of weak limits comes from the fact that, together with Example 1.7, Theorem 1.1 provides for a particularly simple proof of the following theorem of Khrushchev. 
Theorem 1.9 (Khrushchev [18]). Let $\mathcal{C}$ be a CMV matrix with Verblunsky coefficients $\left\{\alpha_{n}\right\}_{n=0}^{\infty}$ and measure $\mu$, and let $d \mu_{n}(\theta)=\left|\varphi_{n}\left(e^{i \theta}\right)\right|^{2} d \mu(\theta)$. Then

$$
d \mu_{n}(\theta) \rightarrow \frac{d \theta}{2 \pi}
$$

weakly if and only if

$$
\forall k \geq 1 \lim _{n \rightarrow \infty} \alpha_{n} \alpha_{n+k}=0 .
$$

Furthermore, these conditions imply that either $\alpha_{n} \rightarrow 0$ or $\mu$ is purely singular.

This theorem is naturally a part of a larger discussion dealing with weak limits of $\mu_{n}$. In particular, Khrushchev's theory deals with the cases in which such weak limits exist. We will show that the analysis of these cases becomes simple when performed using right limits. The reason for this is that the $\mu_{n}$ above are actually the spectral measures of $\mathcal{C}$ and $\delta_{n}$ and, along a proper subsequence, these converge weakly to the corresponding spectral measures of the right limit. Thus, if $\mu_{n}$ converges weakly to $\nu$ as $n \rightarrow \infty$, all of the diagonal measures of any right limit are $\nu$. This leads naturally to

Definition 1.10. We say that a whole-line CMV matrix, $\mathcal{E}$, belongs to Khrushchev Class if $\mu_{n}=\mu_{m}$ for all $n, m \in \mathbb{Z}$, where $\mu_{j}$ is the spectral measure of $\mathcal{E}$ and $\delta_{j}$.

By the discussion above,

Proposition 1.11. If $\mathcal{C}$ is a $C M V$ matrix such that the sequence $\mu_{n}$ has a weak limit as $n \rightarrow \infty$ then all right limits of $\mathcal{C}$ belong to Khrushchev Class.

Thus, Khrushchev theory reduces to the analysis of Khrushchev Class. Since Simon analyzed the analogous Jacobi case [35], we feel the following is fitting:

Definition 1.12. We say that a whole-line Jacobi matrix, $H$, belongs to Simon Class if $\mu_{n}=\mu_{m}$ for all $n, m \in \mathbb{Z}$, where $\mu_{j}$ is the spectral measure of $H$ and $\delta_{j}$.

The final section of this paper will be devoted to the analysis of these two classes. In particular, we rederive all of the main results of [35] and even extend some of those of [19]. We conclude with an amusing (and easy) fact:

Proposition 1.13. Any $H$ in the Simon Class is either periodic, and so reflectionless on its spectrum, or decomposes into a direct sum of finite (in fact $2 \times 2$ matrices), and so has pure point spectrum of infinite multiplicity.

Similarly, any $\mathcal{E}$ in the Khrushchev Class that does not belong to the class introduced in Example 1.7 is either reflectionless on its spectrum or has pure point spectrum.

The rest of this paper is structured as follows. Section 2 contains the proof of Theorems 1.4 and 1.1 as well as an application to random perturbations of CMV matrices. Section 3 contains a proof of Theorems 1.8 and 1.9, and Section 4 contains our analysis of the operators in the Khrushchev and Simon Classes and their relevance to Khrushchev's theory of weak limits and ratio asymptotics.

Acknowledgments. We would like to thank Barry Simon for helpful discussions, as well as the referees for their useful comments. 


\section{The Proof of Remling's Theorem for OPUC}

Our proof will parallel that of Remling [33] quite closely, so we will content ourselves with presenting the parts that differ significantly, but only sketching those parts that are similar.

We will first need some definitions. Let $z \in \mathbb{D}$ and let $S \subset \partial \mathbb{D}$ a Borel set, and define

$$
\omega_{z}(S)=\int_{S} \operatorname{Re}\left(\frac{e^{i \theta}+z}{e^{i \theta}-z}\right) \frac{d \theta}{2 \pi} .
$$

(Here, and numerous times below, we have made use of the standard identification of $\partial \mathbb{D}$ with $[0,2 \pi)$ in that the integration is actually over the set $\left\{\theta \in[0,2 \pi): e^{i \theta} \in S\right\}$. We trust this will not cause any confusion.) If $f: \mathbb{D} \rightarrow \mathbb{D}$ is a Schur function, define

$$
\omega_{f\left(e^{i \theta}\right)}(S)=\lim _{r \uparrow 1} \omega_{f\left(r e^{i \theta}\right)}(S) .
$$

As $z \mapsto \omega_{f(z)}(S)$ is a non-negative harmonic function in $\mathbb{D}$, Fatou's Theorem implies that this limit exists for (Lebesgue) almost every $\theta$.

Given Schur functions $f_{n}(z)$ and $f(z)$, we will say that $f_{n}$ converges to $f$ in the sense of Pearson if for all Borel sets $A, S \subseteq \partial \mathbb{D}$,

$$
\lim _{n \rightarrow \infty} \int_{A} \omega_{f_{n}\left(e^{i \theta}\right)}(S) \frac{d \theta}{2 \pi}=\int_{A} \omega_{f\left(e^{i \theta}\right)}(S) \frac{d \theta}{2 \pi} .
$$

(We note here that in $[1,2,33]$ this mode of convergence was called convergence in value distribution. However, since this term had already been used in [26] for a completely different concept, we will use the above name instead.)

The next lemma relates this type of convergence to a more standard one:

Lemma 2.1. Let $f, f_{n}, n \in \mathbb{N}$, be Schur functions. Then $f_{n}$ converges to $f$ in the sense of Pearson if and only if $f_{n}(z)$ converges to $f(z)$ uniformly on compact subsets of $\mathbb{D}$.

Of course, in this case it is well-known that the associated spectral measures then converge weakly as well.

Proof. We simply sketch the proof since the full details may be found in [33]. For the forward implication, we may use compactness to pick a subsequence where $g(z):=\lim _{k \rightarrow \infty} f_{n_{k}}$ exists (uniformly on compact subsets of $\mathbb{D}$ ) and defines an analytic function. By uniqueness of limits in the sense of Pearson, we then must have $g=f$.

For the opposite direction, one may either use spectral averaging (as in [33]), or simply appeal to Lemma 2.4 below.

The basic result behind Theorem 1.4 is the following analog of a result of Breimesser and Pearson [1]:

Theorem 2.2. Let $\mathcal{C}$ be a half-line $C M V$ matrix. For all Borel sets $S \subseteq \partial \mathbb{D}$ and $A \subseteq \Sigma_{\mathrm{ac}}(\mathcal{C})$ we have

$$
\lim _{n \rightarrow \infty}\left(\int_{A} \omega_{f_{+}\left(e^{i \theta}\right)}(S) \frac{d \theta}{2 \pi}-\int_{A} \omega_{e^{i \theta} f_{-}\left(e^{i \theta}\right)}\left(S^{*}\right) \frac{d \theta}{2 \pi}\right)=0
$$

where $S^{*}=\{z: \bar{z} \in S\}$.

Assuming Theorem 2.2 for a moment, we can prove Theorem 1.4: 
Proof of Theorem 1.4. Let $\mathcal{E}$ be a right limit of $\mathcal{C}$, so there is a sequence $n_{j} \uparrow \infty$ such that $\lim _{j \rightarrow \infty} \alpha_{n+n_{j}}(\mathcal{C})=\alpha_{n}(\mathcal{E})$ for the corresponding sequences of Verblunsky coefficients. Thus, if $f_{ \pm}(z)$ are the Schur functions of $\mathcal{E}$ defined by (1.7) for $n=0$, then

$$
f_{ \pm}\left(z, n_{j}\right) \rightarrow f_{ \pm}(z) \text { as } j \rightarrow \infty
$$

uniformly on compact subsets of $\mathbb{D}$. By Lemma 2.1 and Theorem 2.2 we now have

$$
\int_{A} \omega_{f_{+}\left(e^{i \theta}\right)}(S) \frac{d \theta}{2 \pi}=\int_{A} \omega_{e^{i \theta} f_{-}\left(e^{i \theta}\right)}\left(S^{*}\right) \frac{d \theta}{2 \pi}
$$

for all Borel sets $A \subseteq \Sigma_{\text {ac }}(\mathcal{C}), S \subseteq \partial \mathbb{D}$.

Now Lebesgue's differentiation theorem and the fact that $\omega_{\bar{z}}\left(S^{*}\right)=\omega_{z}(S)$ shows

$$
f_{+}\left(e^{i \theta}\right)=e^{-i \theta} \overline{f_{-}\left(e^{i \theta}\right)}
$$

almost everywhere on $\Sigma_{\text {ac }}(\mathcal{C})$. Thus, $\mathcal{E}$ is reflectionless on $\Sigma_{\text {ac }}(\mathcal{C})$.

We now turn to the proof of Theorem 2.2. We will need a few preparatory results.

Lemma 2.3. For any Schur function $f(z)$, Borel set $S \subseteq \partial \mathbb{D}$, and $z \in \mathbb{D}$ we have

$$
\omega_{f(z)}(S)=\int_{0}^{2 \pi} \omega_{f\left(e^{i \theta}\right)}(S) d \omega_{z}\left(e^{i \theta}\right) .
$$

In particular, for any Borel set $A \subseteq \partial \mathbb{D}$,

$$
\int_{A} \omega_{f\left(r e^{i \theta}\right)}(S) \frac{d \theta}{2 \pi}=\int_{0}^{2 \pi} \omega_{f\left(e^{i \theta}\right)}(S) \omega_{r e^{i \theta}}(A) \frac{d \theta}{2 \pi} .
$$

Proof. For the first statement, just note that both sides are harmonic functions of $z$ with the same boundary values. The second statement follows by writing

$$
d \omega_{r e^{i \theta}}\left(e^{i \phi}\right)=\frac{1-r^{2}}{1+r^{2}-2 r \cos (\phi-\theta)} \frac{d \phi}{2 \pi}
$$

and applying Fubini's theorem.

Lemma 2.4. Let $A \subseteq \partial \mathbb{D}$ be a Borel subset. Then

$$
\limsup _{r \uparrow 1}\left|\int_{f, S} \omega_{f\left(r e^{i \theta}\right)}(S) \frac{d \theta}{2 \pi}-\int_{A} \omega_{f\left(e^{i \theta}\right)}(S) \frac{d \theta}{2 \pi}\right|=0
$$

where the supremum is taken over all Schur functions $f(z)$ and all Borel sets $S \subseteq$ $\partial \mathbb{D}$.

Proof. This follows from Lemma 2.3 and analyzing (the $f$-independent quantity)

$$
\int_{A} \omega_{r e^{i \theta}}\left(A^{c}\right) \frac{d \theta}{2 \pi}
$$

For more details, see Lemma A.1 in [33] whose proof is nearly identical.

We will need a notion of pseudohyperbolic distance on $\mathbb{D}$. Given $w_{1}, w_{2} \in \mathbb{D}$ define

$$
\gamma\left(w_{1}, w_{2}\right)=\frac{\left|w_{1}-w_{2}\right|}{\sqrt{1-\left|w_{1}\right|^{2}} \sqrt{1-\left|w_{2}\right|^{2}}} .
$$

This is an increasing function of the hyperbolic distance on $\mathbb{D}$. As such, if $F: \mathbb{D} \rightarrow \mathbb{D}$ is analytic, then

$$
\gamma\left(F\left(w_{1}\right), F\left(w_{2}\right)\right) \leq \gamma\left(w_{1}, w_{2}\right)
$$


and if $F$ is an automorphism with respect to hyperbolic distance on $\mathbb{D}$ (written " $F \in$ $\operatorname{Aut}(\mathbb{D})$ ") then we have equality above. Taking $F(z)$ to be the analytic function whose real part is $\omega_{z}(S)$, we see that for all $z, \zeta \in \mathbb{D}$ and all Borel sets $S \subseteq \partial \mathbb{D}$,

$$
\left|\omega_{z}(S)-\omega_{\zeta}(S)\right| \leq \frac{\left|\omega_{z}(S)-\omega_{\zeta}(S)\right|}{\sqrt{1-\left|\omega_{z}(S)\right|^{2}} \sqrt{1-\left|\omega_{\zeta}(S)\right|^{2}}} \leq \gamma(F(z), F(\zeta)) \leq \gamma(z, \zeta)
$$

Now let $\left\{\alpha_{n}\right\}_{n \in \mathbb{Z}}$ be a sequence of Verblunsky coefficients (some of which may lie on $\partial \mathbb{D})$. Recall the two sequences of Schur functions defined by (1.7):

$$
f_{+}(z, n)=f\left(z ; \alpha_{n}, \alpha_{n+1}, \ldots\right), \quad f_{-}(z, n)=f\left(z ;-\overline{\alpha_{n-1}},-\overline{\alpha_{n-2}}, \ldots\right) .
$$

Since the Schur algorithm terminates at any $\alpha_{k} \in \partial \mathbb{D}$, we see that for a half-line sequence of $\alpha$ 's (recall $\alpha_{-1}=-1$ ) we have $f_{-}(z, n=0)=-\overline{\alpha_{-1}}=1$.

Viewing matrix arithmetic projectively (that is, identifying an automorphism of $\mathbb{D}$ with its coefficient matrix, see for instance [33]), the Schur algorithm shows

$$
f_{ \pm}(z, n+1)=T_{ \pm}\left(z, \alpha_{n}\right) f_{ \pm}(z, n)
$$

where

$$
T_{+}(z, \alpha)=\left[\begin{array}{cc}
1 & -\alpha \\
-z \bar{\alpha} & z
\end{array}\right] \text { and } T_{-}(z, \alpha)=\left[\begin{array}{cc}
z & -\bar{\alpha} \\
-z \alpha & 1
\end{array}\right] .
$$

By elementary manipulations we see that for any $z \in \mathbb{C}$,

$$
T_{+}(z, \alpha)=\left[\begin{array}{cc}
1 & 0 \\
0 & z
\end{array}\right] \overline{T_{-}(z, \alpha)}\left[\begin{array}{cc}
1 & 0 \\
0 & \bar{z}
\end{array}\right] .
$$

We will let

$$
P_{ \pm}(z, n)=T_{ \pm}\left(z, \alpha_{n-1}\right) \cdots T_{ \pm}\left(z, \alpha_{0}\right)
$$

so that

$$
f_{ \pm}(z, n)=P_{ \pm}(z, n) f_{ \pm}(z, n=0) .
$$

We have the following mapping properties of $T_{ \pm}(z, \alpha)$ :

Lemma 2.5. Let $\alpha \in \mathbb{D}$.

(1) If $z \in \partial \mathbb{D}$, then $T_{ \pm}(z, \alpha) \in \operatorname{Aut}(\mathbb{D})$.

(2) If $z \in \mathbb{D}$, then $T_{-}(z, \alpha): \mathbb{D} \rightarrow \mathbb{D}$ and

$$
\gamma\left(T_{-}(z, \alpha) w_{1}, T_{-}(z, \alpha) w_{2}\right) \leq|z| \gamma\left(w_{1}, w_{2}\right)
$$

for all $w_{1}, w_{2} \in \mathbb{D}$.

Proof. Let

so that

$$
S(\alpha)=\left[\begin{array}{cc}
1 & -\alpha \\
-\bar{\alpha} & 1
\end{array}\right] \quad \text { and } \quad M(z)=\left[\begin{array}{cc}
z & 0 \\
0 & 1
\end{array}\right]
$$

$$
T_{+}(z, \alpha)=M\left(z^{-1}\right) S(\alpha) \quad \text { and } \quad T_{-}(z, \alpha)=S(\bar{\alpha}) M(z) .
$$

Because $\alpha \in \mathbb{D}$ we have $S(\alpha) \in \operatorname{Aut}(\mathbb{D})$. If $z \in \partial \mathbb{D}$ then $M(z) \in \operatorname{Aut}(\mathbb{D})$ as well, while a straightforward calculation shows that if $z \in \mathbb{D}$ then

$$
\gamma\left(M(z) w_{1}, M(z) w_{2}\right) \leq|z| \gamma\left(w_{1}, w_{2}\right) .
$$

This proves (1) and (2).

With these preliminaries in hand we are ready for the proof of Theorem 2.2. We emphasize again that we are following the proof of Theorem 3.1 from [33].

Proof of Theorem 2.2. Subdivide $A=A_{0} \cup A_{1} \cup \cdots \cup A_{N}$ in such a way that 
1. $\left|A_{0}\right|<\varepsilon$.

2. On $\bigcup_{k=1}^{N} A_{k}, \lim _{r \uparrow 1} f_{+}\left(r e^{i \theta}, 0\right)$ exists and lies in $\mathbb{D}$.

3. For each $1 \leq k \leq N$, there is a point $m_{k} \in \mathbb{D}$ such that $\gamma\left(f_{+}\left(e^{i \theta}, 0\right), m_{k}\right)<\varepsilon$ for all $e^{i \theta} \in A_{k}$.

The construction of such a decomposition is identical to that given in [33], so we do not review it here.

To deal with $A_{0}$, we note that for any $z \in \overline{\mathbb{D}}$ and any Borel set $S \subseteq \partial \mathbb{D}$, we have $\left|\omega_{z}(S)\right| \leq 1$. Thus

$$
\left|\int_{A_{0}} \omega_{f_{+}\left(e^{i \theta}, n\right)}(S) \frac{d \theta}{2 \pi}-\int_{A_{0}} \omega_{e^{i \theta} f_{-}\left(e^{i \theta}, n\right)}\left(S^{*}\right) \frac{d \theta}{2 \pi}\right|<2 \varepsilon .
$$

Now we consider $A_{1}, \ldots, A_{N}$. Notice that if $e^{i \theta} \in \bigcup_{k=1}^{N} A_{k}$, then for all $n \in \mathbb{N}$ we also have that $\lim _{r \uparrow 1} f_{+}\left(r e^{i \theta}, n\right)$ exists and lies in $\mathbb{D}$. As $P_{+}\left(e^{i \theta}, n\right) \in \operatorname{Aut}(\mathbb{D})$ we see

$$
\gamma\left(f_{+}\left(e^{i \theta}, n\right), P_{+}\left(e^{i \theta}, n\right) m_{k}\right)<\varepsilon
$$

for all $e^{i \theta} \in A_{k}$ and all $n \in \mathbb{N}$. Using (2.1) and integrating we find

$$
\left|\int_{A_{k}} \omega_{f_{+}\left(e^{i \theta}, n\right)}(S) \frac{d \theta}{2 \pi}-\int_{A_{k}} \omega_{P_{+}\left(e^{i \theta}, n\right) m_{k}}(S) \frac{d \theta}{2 \pi}\right|<\varepsilon\left|A_{k}\right| .
$$

By $(2.2)$ and the fact that $\omega_{\bar{z}}\left(S^{*}\right)=\omega_{z}(S)$, we can rewrite this as

$$
\left|\int_{A_{k}} \omega_{f_{+}\left(e^{i \theta}, n\right)}(S) \frac{d \theta}{2 \pi}-\int_{A_{k}} \omega_{e^{i \theta} P_{-}\left(e^{i \theta}, n\right)\left(e^{-i \theta} m_{k}\right)}\left(S^{*}\right) \frac{d \theta}{2 \pi}\right|<\varepsilon\left|A_{k}\right|
$$

(and notice that because $T_{-}(z, \alpha)=S(\bar{\alpha}) M(z)$, we have that $z P_{-}(z, n)\left(z^{-1} m_{k}\right)$ is indeed a Schur function).

By Lemma 2.5 there is an $n_{0} \in \mathbb{N}$ so that for all $n \geq n_{0}$,

$$
\gamma\left(z P_{-}(z, n)\left(z^{-1} w_{k}\right), z f_{-}(z, n)\right)<\varepsilon .
$$

As before, using (2.1) and integrating shows

$$
\left|\int_{A_{k}} \omega_{z P_{-}(z, n)\left(z^{-1} w_{k}\right)}\left(S^{*}\right) \frac{d \theta}{2 \pi}-\int_{A_{k}} \omega_{z f_{-}(z, n)}\left(S^{*}\right) \frac{d \theta}{2 \pi}\right|<\varepsilon\left|A_{k}\right| .
$$

Now use Lemma 2.4 to find an $r<1$ so that

$$
\left|\int_{A_{k}} \omega_{f\left(e^{i \theta}\right)}(S) \frac{d \theta}{2 \pi}-\int_{A_{k}} \omega_{f\left(r e^{i \theta}\right)}(S) \frac{d \theta}{2 \pi}\right|<\varepsilon\left|A_{k}\right|
$$

for all Schur functions $f(z)$, all Borel sets $S \subseteq \partial \mathbb{D}$, and $k=1, \ldots, N$. Applying this to (2.3) and (2.4) shows

$$
\left|\int_{A_{k}} \omega_{f_{+}\left(e^{i \theta}, n\right)}(S) \frac{d \theta}{2 \pi}-\int_{A_{k}} \omega_{e^{i \theta} f_{-}\left(e^{i \theta}, n\right)}\left(S^{*}\right) \frac{d \theta}{2 \pi}\right|<4 \varepsilon\left|A_{k}\right| .
$$

Now summing in $k$ shows

$$
\left|\int_{A} \omega_{f_{+}\left(e^{i \theta}, n\right)}(S) \frac{d \theta}{2 \pi}-\int_{A} \omega_{e^{i \theta} f_{-}\left(e^{i \theta}, n\right)}\left(S^{*}\right) \frac{d \theta}{2 \pi}\right|<4 \varepsilon|A|+2 \varepsilon
$$

for all $n \geq n_{0}$. 
Next, we illustrate Theorem 1.4 by a simple example of constant coefficients CMV matrices:

Example 2.6. Let $\mathcal{C}$ be the half-line CMV matrix associated with the constant Verblunsky coefficients $\alpha_{n}=a, n \geq 0$, for some $a \in(0,1)$. It follows from the Schur algorithm that the corresponding Schur function $f_{a}$ satisfies the quadratic equation

$$
a z f_{a}(z)^{2}+(1-z) f_{a}(z)-a=0,
$$

and hence is given by

$$
f_{a}(z)=\frac{-(1-z)+\sqrt{(1-z)^{2}+4 a^{2} z}}{2 a z}, \quad z \in \mathbb{D},
$$

where the square root is defined so that $\sqrt{e^{i \theta}}=e^{i \theta / 2}$ for $\theta \in(-\pi, \pi)$. Using the Carathéodory function $F_{a}(z)=\frac{1+z f_{a}(z)}{1-z f_{a}(z)}$ we compute

$$
\begin{aligned}
\Sigma_{\mathrm{ac}}(\mathcal{C}) & =\left\{e^{i \theta}: \operatorname{Re} F_{a}\left(e^{i \theta}\right)>0\right\}=\left\{e^{i \theta}:\left|f_{a}\left(e^{i \theta}\right)\right|<1\right\} \\
& =\left\{e^{i \theta}: 2 \arcsin (a)<\theta<2 \pi-2 \arcsin (a)\right\} .
\end{aligned}
$$

The half-line CMV matrix $\mathcal{C}$ has exactly one right limit $\mathcal{E}$ which is the whole-line CMV matrix associated with the constant coefficients $\widetilde{\alpha}_{n}=a, n \in \mathbb{Z}$. It follows from (1.7) that the two Schur functions for $\mathcal{E}$ are given by $f_{+}(z, n)=f_{a}(z)$ and $f_{-}(z, n)=f_{-a}(z)=-f_{a}(z), n \in \mathbb{Z}$. Since for all $e^{i \theta} \in \Sigma_{\mathrm{ac}}(\mathcal{C})$,

$$
f_{a}\left(e^{i \theta}\right)=\frac{i \sin (\theta / 2)}{a e^{i \theta / 2}}\left(1-\sqrt{1-\left(\frac{a}{\sin (\theta / 2)}\right)^{2}}\right)
$$

and the expression under the square root is positive, one easily verifies the reflectionless property of $\mathcal{E}$ on $\Sigma_{\mathrm{ac}}(\mathcal{C})$,

$$
e^{i \theta} f_{+}\left(e^{i \theta}, n\right)=e^{i \theta} f_{a}\left(e^{i \theta}\right)=-\overline{f_{a}\left(e^{i \theta}\right)}=\overline{f_{-}\left(e^{i \theta}, n\right)}, \quad e^{i \theta} \in \Sigma_{\mathrm{ac}}(\mathcal{C}),
$$

thus confirming the claim of Theorem 1.4.

Note that adding a decaying perturbation to the Verblunsky coefficients of $\mathcal{C}$ does not change the uniqueness of the right limit, nor does it change the limiting operator. Moreover, if the decay is sufficiently fast (e.g. $\left.\ell^{1}\right), \Sigma_{\mathrm{ac}}(\mathcal{C})$ does not change either.

The following is one of the reasons reflectionless operators are so useful:

Lemma 2.7. Let $\left\{\alpha_{n}\right\}_{n \in \mathbb{Z}},\left\{\beta_{n}\right\}_{n \in \mathbb{Z}}$ be two sequences of Verblunsky coefficients such that their corresponding whole-line CMV matrices are both reflectionless on some common set $A$ with $|A|>0$. If $\alpha_{n}=\beta_{n}$ for all $n<0$, then $\alpha_{n}=\beta_{n}$ for all $n$.

Proof. By the Schur algorithm, $\left\{\alpha_{n}\right\}_{n<0}$ determines $f_{-}(z, 0)$. This, by Definition 1.3 , determines $f_{+}(z, 0)$ on $A$. But the values of a Schur function on a set of positive Lebesgue measure on $\partial \mathbb{D}$ determine the Schur function. Thus, $f_{+}(z, 0)$ is determined throughout $\mathbb{D}$ by $\left\{\alpha_{n}\right\}_{n<0}$. But, by the Schur algorithm again, this determines $\left\{\alpha_{n}\right\}_{n \geq 0}$.

Proof of Theorem 1.1. Take a subsequence $\left\{n_{j_{k}}\right\}_{k=1}^{\infty}$, of $n_{j}$, such that both

$$
\lim _{k \rightarrow \infty} \alpha_{n+n_{j_{k}}} \equiv \beta_{n}
$$


and

$$
\lim _{k \rightarrow \infty} \widetilde{\alpha}_{n+n_{j_{k}}} \equiv \widetilde{\beta}_{n}
$$

exist for every $n \in \mathbb{Z}$. By conditions (ii) and (iii) of the theorem, $\beta_{n}=\widetilde{\beta}_{n}$ for all $n<0$ but $\beta_{0} \neq \widetilde{\beta}_{0}$. By Theorem 1.4 the corresponding whole-line CMV matrices, $\mathcal{E}$ and $\widetilde{\mathcal{E}}$, are reflectionless on $\Sigma_{\text {ac }}(\mathcal{C})$ and $\Sigma_{\text {ac }}(\widetilde{\mathcal{C}})$ respectively. Thus, by Lemma 2.7 these two sets cannot intersect each other.

Viewing a CMV matrix satisfying (1.4) as a perturbation of the CMV matrix with all Verblunsky coefficients equal to zero (this matrix has spectral measure $\frac{d \theta}{2 \pi}$ ), we see by the above analysis that such a matrix cannot have any absolutely continuous spectrum, since (1.4) is easily seen to imply conditions (ii) and (iii) of the theorem.

We conclude this section with an application of Theorem 1.4 to random CMV matrices.

Theorem 2.8. Let $\left\{\beta_{n}(\omega)\right\}_{n=1}^{\infty}$ be a sequence of random Verblunsky coefficients of the form:

$$
\beta_{n}(\omega)=\alpha_{n}+s_{n} X_{n}(\omega)
$$

where $X_{n}(\omega)$ is a sequence of independent, identically distributed random variables whose common distribution is not supported at a single point, and $s_{n}$ is a bounded sequence such that $\left|\alpha_{n}+s_{n}\right|<1$. Let $\mathcal{C}(\omega)$ be the corresponding random $C M V$ matrix. If $s_{n} \not \rightarrow 0$ as $n \rightarrow \infty$ then $\Sigma_{\mathrm{ac}}(\mathcal{C}(\omega))=\emptyset$ almost surely.

We first need a lemma:

Lemma 2.9. Let $\left\{\beta_{n}(\omega)\right\}_{n=1}^{\infty}$ be a sequence of independent random Verblunsky coefficients and let $\mathcal{C}(\omega)$ be the corresponding family of $C M V$ matrices. Then there exists a set $A \subseteq \partial \mathbb{D}$ such that with probability one, $\Sigma_{\mathrm{ac}}(\mathcal{C}(\omega))=A$.

Proof. This is the CMV version of a theorem of Jakšić and Last [16, Cor. 1.1.3] for Jacobi matrices. The proof is the same: Since the absolutely continuous spectrum is stable under finite rank perturbations, it is easily seen to be a tail event. Thus, the result is implied by Kolmogorov's 0-1 Law. For details see [16].

Proof of Theorem 2.8. Pick a sequence, $n_{j}$, such that $\lim _{j \rightarrow \infty} s_{n+n_{j}} \equiv S_{n}$ exists for every $n \in \mathbb{Z}$ and $S_{0} \neq 0$. Such a sequence exists by the assumptions on $s_{n}$. By restricting to a subsequence, we may assume that $\lim _{j \rightarrow \infty} \alpha_{n+n_{j}}+s_{n+n_{j}}=\widetilde{\alpha}_{n}+S_{n}$ also exists for any $n \in \mathbb{Z}$.

Let $X_{1} \neq X_{2}$ be two points in the support of the common distribution of $X_{n}(\omega)$. By the Borel-Cantelli Lemma, with probability one, there exist two subsequences $n_{j_{k}(\omega)}$ and $n_{j_{l}(\omega)}$, such that

$$
\lim _{k \rightarrow \infty} X_{n+n_{j_{k}(\omega)}}(\omega)=X_{1}, \quad n \in \mathbb{Z}
$$

and

$$
\lim _{l \rightarrow \infty} X_{n+n_{j_{l}(\omega)}}(\omega)= \begin{cases}X_{1} & n<0 \\ X_{2} & n \geq 0\end{cases}
$$

Let $\mathcal{E}_{1}$ and $\mathcal{E}_{2}$ be the two whole-line CMV matrices corresponding to the sequences

$$
\widetilde{\beta}_{n}^{1} \equiv \widetilde{\alpha}_{n}+S_{n} X_{1}, \quad n \in \mathbb{Z}
$$


and

$$
\widetilde{\beta}_{n}^{2}= \begin{cases}\widetilde{\alpha}_{n}+S_{n} X_{1} & n<0, \\ \widetilde{\alpha}_{n}+S_{n} X_{2} & n \geq 0 .\end{cases}
$$

respectively. If it were not true that $\Sigma_{\mathrm{ac}}(\mathcal{C}(\omega))=\emptyset$ almost surely, then by Lemma 2.9 , the essential support of the absolutely continuous spectrum would be some deterministic set $A \neq \emptyset$. By Theorem 1.4, since $\mathcal{E}_{1}$ and $\mathcal{E}_{2}$ are both right limits of CMV matrices with absolutely continuous spectrum on $A$, they are both reflectionless on $A$. This contradicts Lemma 2.7 so we see that $\Sigma_{\text {ac }}(\mathcal{C}(\omega))=\emptyset$ almost surely.

\section{Reflectionless Matrices and Reflectionless Measures}

In this section we verify that Example 1.7 is the only example where the two notions of reflectionless (cf. Definitions 1.3 and 1.6) are not equivalent. As an application of this fact we give a short proof of Theorem 1.9, a special case of Khrushchev's results.

We start by introducing the whole-line unitary 5-diagonal CMV matrix $\mathcal{E}$ associated to $\left\{\alpha_{n}\right\}_{n \in \mathbb{Z}}$ by

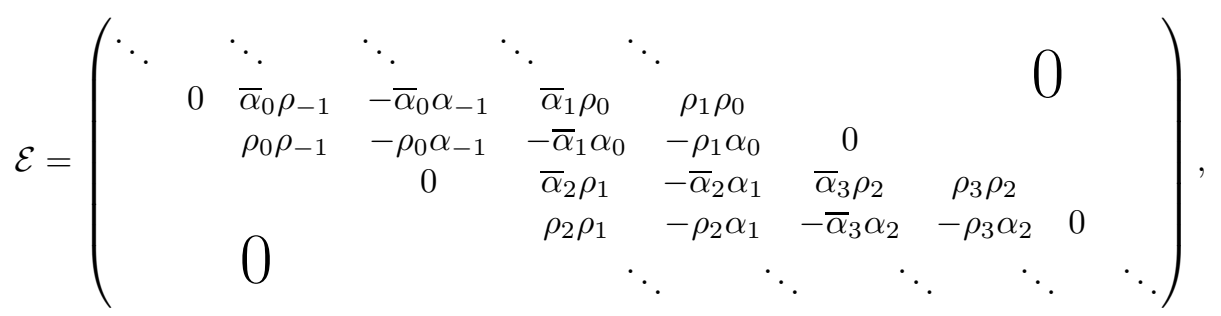

where $\rho_{n}=\left(1-\left|\alpha_{n}\right|^{2}\right)^{1 / 2}$. Here the diagonal elements are given by $\mathcal{E}_{n, n}=$ $-\bar{\alpha}_{n} \alpha_{n-1}$. It is known $[4,36]$ that $\mathrm{CMV}$ matrices have the following $\mathcal{L} \mathcal{M}$ factorization:

$$
\mathcal{E}=\mathcal{L} \mathcal{M}
$$

where

$$
\mathcal{L}=\left(\begin{array}{llll}
\ddots & & & 0 \\
& \theta_{2 k-2} & & \\
0 & \theta_{2 k} & \\
0 & & \ddots
\end{array}\right), \quad \mathcal{M}=\left(\begin{array}{cccc}
\ddots & & \\
& \theta_{2 k-1} & 0 \\
0 & \theta_{2 k+1} & \\
0 & & \ddots
\end{array}\right),
$$

such that, for all $k \in \mathbb{Z}$

$$
\begin{gathered}
\left(\begin{array}{cc}
\mathcal{L}_{2 k, 2 k} & \mathcal{L}_{2 k, 2 k+1} \\
\mathcal{L}_{2 k+1,2 k} & \mathcal{L}_{2 k+1,2 k+1}
\end{array}\right)=\theta_{2 k}, \quad\left(\begin{array}{cc}
\mathcal{M}_{2 k-1,2 k-1} & \mathcal{M}_{2 k-1,2 k} \\
\mathcal{M}_{2 k, 2 k-1} & \mathcal{M}_{2 k, 2 k}
\end{array}\right)=\theta_{2 k-1} \\
\theta_{k}=\left(\begin{array}{cc}
\bar{\alpha}_{k} & \rho_{k} \\
\rho_{k} & -\alpha_{k}
\end{array}\right) .
\end{gathered}
$$


Next, we introduce the diagonal Schur function $f(z, n)$ associated with the diagonal spectral measure $\mu_{n}$ (i.e., the spectral measure of $\mathcal{E}$ and $\delta_{n}$ ) by

$$
\frac{1+z f(z, n)}{1-z f(z, n)}=\int_{0}^{2 \pi} \frac{e^{i \theta}+z}{e^{i \theta}-z} d \mu_{n}(\theta)=\left\langle\delta_{n},(\mathcal{E}+z I)(\mathcal{E}-z I)^{-1} \delta_{n}\right\rangle, \quad n \in \mathbb{Z} .
$$

Then it follows from Definition 1.6 that the measure $\mu_{n}$ is reflectionless on $A \subseteq \partial \mathbb{D}$ if and only if $\operatorname{Im}(z f(z, n))=0$ for a.e. $z \in A$. Recall from (1.9) that the diagonal and half-line Schur functions are related by

$$
f(z, n)=f_{+}(z, n) f_{-}(z, n) .
$$

Thus, if a whole-line CMV matrix, $\mathcal{E}$, is reflectionless on $A$, it follows that all diagonal measures $\mu_{n}, n \in \mathbb{Z}$, are reflectionless on $A$ as well. As indicated in Example 1.7, the converse is not true in general. Nevertheless, one can show that this example is the only exceptional case: whenever all $\alpha$ 's are identically zero, or there are at least two nonzero $\alpha$ 's, the converse holds. We start by showing that a reflectionless CMV matrix $\mathcal{E}$ with a finite number of nonzero $\alpha$ 's can have no more than one nonzero Verblunsky coefficient.

Lemma 3.1. Suppose $\mathcal{E}$ is a whole-line $C M V$ matrix of the form (3.1) such that $\alpha_{m} \neq 0, \alpha_{n} \neq 0$ for some $m, n \in \mathbb{Z}, m<n$, and $\mu_{n}$ is reflectionless on a set $A \subseteq \partial \mathbb{D}$ of positive Lebesgue measure. Then there are infinitely many nonzero $\alpha$ 's.

Proof. Assume that there are finitely many nonzero $\alpha$ 's. Then it follows from the Schur algorithm that the Schur functions $f_{ \pm}(z, n)$ are rational functions of $z$ with finitely many zeros in $\overline{\mathbb{D}}$ and poles in $\mathbb{C} \backslash \overline{\mathbb{D}}$. By $(1.9)$ the same holds for $f(z, n)$.

Let $B$ be a neighborhood of the finite set of zeros of $f(z, n)$ on $\partial \mathbb{D}$ such that $A \backslash B$ has positive Lebesgue measure. Then $\log (z f(z, n))$ is a well-defined analytic function on some open neighborhood of $\partial \mathbb{D} \backslash B$.

The reflectionless assumption implies that $\operatorname{Im} \log (z f(z, n)) \in\{0, \pi\}$ Lebesgue a.e. on $A \backslash B$, and hence by the Cauchy-Riemann equations, the analytic function $\frac{d}{d z} \log (z f(z, n))$ is zero on accumulation points of $A \backslash B$. Since $A \backslash B$ is of positive Lebesgue measure, the set of its accumulation points is also of positive Lebesgue measure, and hence $\frac{d}{d z} \log (z f(z, n))$ is identically zero in the neighborhood of $\partial \mathbb{D} \backslash B$. This implies that $z f(z, n)$ is a nonzero constant in $\mathbb{D} \backslash B$. This is a contradiction since $f(z, n)$ is analytic in $\mathbb{D}$.

Proof of Theorem 1.8. Assume $\mathcal{E}$ is reflectionless in the sense of Definition 1.3. Then it follows from the discussion at the beginning of this section that $\mu_{n}$ is reflectionless for all $n$.

Now assume $\mu_{n}$ is reflectionless in the sense of Definition 1.6 for all $n$. Since two $\alpha$ 's are not zero, it follows from Lemma 3.1 that there are infinitely many nonzero $\alpha$ 's. Let $\alpha_{n_{-1}}, \alpha_{n_{0}}, \alpha_{n_{1}}, \alpha_{n_{2}}$, denote four consecutive nonzero $\alpha$ 's, that is, four non-zero values with possibly some zero values between them. For $n \in \mathbb{Z}$, introduce $g_{+}(z, n)=z f_{+}(z, n)$ and $g_{-}(z, n)=\overline{f_{-}(z, n)}$. Then $g_{ \pm}\left(z, n_{j}\right), j=0,1,2$, are not identically zero functions and it follows from the Schur algorithm that for $z \in \partial \mathbb{D}$ and $n \in \mathbb{Z}$,

$$
g_{ \pm}(z, n-1)=z \frac{g_{ \pm}(z, n)+\alpha_{n-1}}{\bar{\alpha}_{n-1} g_{ \pm}(z, n)+1}, \quad g_{ \pm}(z, n+1)=\frac{g_{ \pm}(z, n) / z-\alpha_{n}}{-\bar{\alpha}_{n} g_{ \pm}(z, n) / z+1}
$$

The reflectionless condition at $n_{1}$ implies $g_{+}\left(z, n_{1}\right) \overline{g_{-}\left(z, n_{1}\right)}=z f\left(z, n_{1}\right) \in \mathbb{R} \backslash\{0\}$ for a.e. $z \in A$, and hence $g_{ \pm}\left(z, n_{1}\right)=s_{ \pm}(z) e^{i t(z)}$ with $s_{ \pm}(z) \in \mathbb{R}$ and $t(z) \in[0, \pi)$ 
for a.e. $z \in A$. In order to check that $\mathcal{E}$ is reflectionless in the sense of Definition 1.3 it remains to check that $s_{+}(z)=s_{-}(z)$ for a.e. $z \in A$.

By construction all $\alpha$ 's between $\alpha_{n_{0}}$ and $\alpha_{n_{1}}$ are zero, hence it follows from (3.3) that

$$
g_{ \pm}\left(z, n_{0}\right)=z^{n_{1}-n_{0}} e^{i t(z)} \frac{s_{ \pm}(z)+e^{-i t(z)} \alpha_{n_{0}}}{\bar{\alpha}_{n_{0}} e^{i t(z)} s_{ \pm}(z)+1} \quad \text { for a.e. } z \in A
$$

Since for every $\gamma \in \mathbb{D} \backslash \mathbb{R}$ the function $h_{\gamma}:[-1,1] \rightarrow\left(-\frac{\pi}{2}, \frac{\pi}{2}\right)$ defined by

$$
h(x)=\arg \left(\bar{\gamma} \frac{x+\gamma}{\bar{\gamma} x+1}\right)
$$

is $1-1,(3.3)$ and the reflectionless condition at $n_{0}$ (i.e., $g_{+}\left(z, n_{0}\right) \overline{g_{-}\left(z, n_{0}\right)}=$ $z f\left(z, n_{0}\right) \in \mathbb{R} \backslash\{0\}$ for a.e. $\left.z \in A\right)$ imply

$$
s_{+}(z)=s_{-}(z) \text { for a.e. } z \in A \text { such that } e^{-i t(z)} \alpha_{n_{0}} \in \mathbb{D} \backslash \mathbb{R} .
$$

Similarly, since by construction all $\alpha$ 's between $\alpha_{n_{1}}$ and $\alpha_{n_{2}}$ are zero, it follows from (3.3) that

$$
g_{ \pm}\left(z, n_{2}\right)=z^{n_{1}-n_{2}} e^{i t(z)} \frac{s_{ \pm}(z)-e^{-i t(z)} z^{n_{2}-n_{1}} \alpha_{n_{1}}}{-\bar{\alpha}_{n_{1}} e^{i t(z)} z^{n_{1}-n_{2}} s_{ \pm}(z)+1} \quad \text { for a.e. } z \in A
$$

and hence, the reflectionless condition at $n_{2}$ (i.e., $g_{+}\left(z, n_{2}\right) \overline{g_{-}\left(z, n_{2}\right)}=z f\left(z, n_{2}\right) \in$ $\mathbb{R} \backslash\{0\}$ for a.e. $z \in A)$, together with the injectivity of $h_{\gamma}$, implies

$$
s_{+}(z)=s_{-}(z) \text { for a.e. } z \in A \text { such that } e^{-i t(z)} z^{n_{2}-n_{1}} \alpha_{n_{2}} \in \mathbb{D} \backslash \mathbb{R} .
$$

Since $e^{-i t(z)} \alpha_{n_{0}} \in \mathbb{R}$ and $e^{-i t(z)} z^{n_{2}-n_{1}} \alpha_{n_{2}} \in \mathbb{R}$ may hold simultaneously only on a finite set, it follows from (3.5) and (3.6) that

$$
s_{+}(z)=s_{-}(z) \text { for a.e. } z \in A,
$$

and hence $g_{+}\left(z, n_{1}\right)=g_{-}\left(z, n_{1}\right)$ for a.e. $z \in A$. That is, $\mathcal{E}$ is reflectionless on $A$ according to Definition 1.3.

Remark. We note that the case of identically zero Verblunsky coefficients corresponds to $f_{ \pm}(z, n) \equiv 0$ for all $n \in \mathbb{Z}$, so that the associated CMV matrix is reflectionless on $\partial \mathbb{D}$ in the sense of Definition 1.3 and hence all its diagonal spectral measures are reflectionless on $\partial \mathbb{D}$ in the sense of Definition 1.6. The case of a single nonzero coefficient, discussed in Example 1.7, corresponds to one of $f_{+}(z, n)$ or $f_{-}(z, n)$ being nonzero and the other being identically zero for each $n \in \mathbb{Z}$, so that the associated CMV matrix is not reflectionless on any subset of $\partial \mathbb{D}$ of positive Lebesgue measure, yet all the diagonal measures are reflectionless on $\partial \mathbb{D}$.

Proof of Theorem 1.9. Let $\mathcal{E}$ be a right limit of $\mathcal{C}$. Then all the diagonal measures of $\mathcal{E}$ are identical and equal to $\frac{d \theta}{2 \pi}$. The corresponding diagonal Schur functions in this case are $f(z, n) \equiv 0$ for all $n \in \mathbb{Z}$. Hence by (1.9) for each $n \in \mathbb{Z}$ either $f_{-}(z, n) \equiv 0$ or $f_{+}(z, n) \equiv 0$ or both. The latter case corresponds to $\mathcal{E}$ having identically zero Verblunsky coefficients and the other two cases correspond to $\mathcal{E}$ having exactly one nonzero Verblunsky coefficient. Since this holds for all right limits of $\mathcal{C}$, we conclude that for all $k \in \mathbb{N}$,

$$
\lim _{n \rightarrow \infty} \alpha_{n} \alpha_{n+k}=0 \text {. }
$$

Conversely, (3.7) implies that all right limits of $\mathcal{C}$ may have at most one nonzero Verblunsky coefficient. Hence all right limits of $\mathcal{C}$ have identical diagonal spectral 
measures equal to $\frac{d \theta}{2 \pi}$. This implies that the diagonal measures $d \mu_{n}$ of $C$ converge weakly to $\frac{d \theta}{2 \pi}$ as $n \rightarrow \infty$.

The final statement follows since if $\alpha_{n} \not \rightarrow 0$ then clearly (1.4) holds, which implies, by Theorem 1.1, that $\mu$ is purely singular.

\section{The Simon and Khrushchev Classes}

In this section we extend the discussion of the previous section to include all cases where $\mu_{n}$ has a weak limit. We consider both the Jacobi and CMV cases, but begin with the Jacobi case since it is technically simpler.

4.1. The Simon Class. A half-line Jacobi matrix is a semi-infinite matrix of the form:

$$
J\left(\left\{a_{n}, b_{n}\right\}_{n=1}^{\infty}\right)=\left(\begin{array}{ccccc}
b_{1} & a_{1} & 0 & 0 & \ldots \\
a_{1} & b_{2} & a_{2} & 0 & \ldots \\
0 & a_{2} & b_{3} & a_{3} & \ldots \\
\ldots & \ldots & \ldots & \ldots & \ldots
\end{array}\right) .
$$

Its whole-line counterpart is defined by

$$
H\left(\left\{a_{n}, b_{n}\right\}_{n \in \mathbb{Z}}\right)=\left(\begin{array}{ccccccc}
\ddots & \ddots & \ddots & & & & 0 \\
& a_{-1} & b_{0} & a_{0} & & & \\
& & a_{0} & b_{1} & a_{1} & & \\
& & & a_{1} & b_{2} & a_{2} & \\
& & & & \ddots & \ddots & \ddots
\end{array}\right),
$$

(we assume $b_{n} \in \mathbb{R}, a_{n} \geq 0$ in both cases).

These matrices may be viewed as operators on $\ell^{2}(\mathbb{N})$ and $\ell^{2}(\mathbb{Z})$, respectively, and both are clearly symmetric. As is well known, the theory of half-line Jacobi matrices with $a_{n}>0$ is intimately related to that of orthogonal polynomials on the real line (OPRL) through the recursion formula for the polynomials (see e.g. [34]). In particular, in the self-adjoint case (to which we shall henceforth restrict our discussion), $\mu$-the spectral measure for $J$ and $\delta_{1}$-is the unique solution to the corresponding moment problem. Furthermore, $d \mu_{n}(x)=\left|p_{n-1}(x)\right|^{2} d \mu(x)$ is the spectral measure for $J$ and $\delta_{n}$, where $\left\{p_{n}(x)\right\}$ are the orthonormal polynomials corresponding to $\mu$. The whole-line matrices enter naturally into this framework as right limits (see e.g. [33] for the definition).

The problem at the center of our discussion is that of the identification of right limits of half-line Jacobi matrices with the property that $\mu_{n}$ has a weak limit as $n \rightarrow \infty$. As is clear from the discussion in the introduction, all these right limits belong to Simon Class (recall Definition 1.12).

Theorem 4.1. Let $H\left(\left\{a_{n}, b_{n}\right\}_{n \in \mathbb{Z}}\right)$ be a whole-line Jacobi matrix. The following are equivalent:

(i) H belongs to Simon Class.

(ii) For all $m, n \in \mathbb{Z}, \int x d \mu_{n}(x)=\int x d \mu_{m}(x)$ and $\int x^{2} d \mu_{n}(x)=\int x^{2} d \mu_{m}(x)$.

(iii) $a_{2 n}=a, a_{2 n+1}=c, b_{n}=b$ for some numbers, $a, c \geq 0$ and $b \in \mathbb{R}$.

Remark. In particular, this shows that if $H$ has constant first and second moments, then $H$ belongs to Simon Class. Note, however, that the values of these moments do not determine the element of the class itself (not even up to translation; see (4.3) and (4.4) below). Thus, it makes sense to define $\mathcal{S}(A, B)$ to be the set of all 
matrices in the Simon Class having $a^{2}+c^{2}=A$ and $b=B$, where $a, b, c$ are as in (iii) above.

Proof. $(i) \Rightarrow(i i)$ is trivial.

$($ ii $) \Rightarrow($ iii $)$ : Noting that

$$
\int x d \mu_{n}(x)=b_{n}
$$

and

$$
\int x^{2} d \mu_{n}(x)=a_{n-1}^{2}+b_{n}^{2}+a_{n}^{2},
$$

the result follows immediately for the diagonal elements. With this in hand, comparing the second moment of $\mu_{n}$ and $\mu_{n+1}$ we see that $a_{n-1}^{2}+a_{n}^{2}=a_{n}^{2}+a_{n+1}^{2}$ from which it follows that $a_{n-1}=a_{n+1}$, and we are done.

(iii) $\Rightarrow\left(\right.$ i): Clearly, by symmetry, $\mu_{n}=\mu_{n+2}$ for all $n$, and if $a=c$ also $\mu_{n}=\mu_{n+1}$. Thus, we are left with showing $\mu_{0}=\mu_{1}$ under the assumption $a \neq c$ (so at least one is nonzero). We shall show that for any $z \in \mathbb{C}_{+}$,

$$
\int \frac{d \mu_{0}(x)}{x-z}=\int \frac{d \mu_{1}(x)}{x-z}
$$

Fix $z \in \mathbb{C}_{+}$and let $\{u(n)\}$ be a sequence satisfying $a_{n} u(n+1)+b_{n} u(n)+$ $a_{n-1} u(n-1)=z u(n)$ that is $\ell^{2}$ at $\infty$. This sequence is unique up to a constant factor. By the symmetry of $H$, note that $v(n) \equiv u(1-n)$ satisfies the same equation and is $\ell^{2}$ at $-\infty$. Now write

$$
\begin{aligned}
\int \frac{d \mu_{0}(x)}{x-z} & =\left\langle\delta_{0},(H-z)^{-1} \delta_{0}\right\rangle=\frac{u(0) v(0)}{v(1) u(0)-v(0) u(1)} \\
& =\frac{u(0) u(1)}{v(1) u(0)-v(0) u(1)}=\frac{v(1) u(1)}{v(1) u(0)-v(0) u(1)} \\
& =\left\langle\delta_{1},(H-z)^{-1} \delta_{1}\right\rangle=\int \frac{d \mu_{1}(x)}{x-z}
\end{aligned}
$$

from which, by standard results, it follows that $\mu_{0}=\mu_{1}$.

We immediately get the following:

Corollary 4.2. Let $J$ be a self-adjoint half-line Jacobi matrix and let $\mu$ be its spectral measure. For $n \geq 1$, let $d \mu_{n}(x)=\left|p_{n-1}(x)\right|^{2} d \mu(x)$ be the spectral measure of $J$ and $\delta_{n}$. If

$$
\begin{aligned}
& \lim _{n \rightarrow \infty} \int x d \mu_{n}(x)=\tilde{B} \\
& \lim _{n \rightarrow \infty} \int x^{2} d \mu_{n}(x)=\tilde{A}
\end{aligned}
$$

then $J$ is bounded and all right limits of $J$ are in $\mathcal{S}\left(\tilde{A}-\tilde{B}^{2}, \tilde{B}\right)$.

Proof. That $J$ is bounded follows from (4.3) and (4.4) applied to $J$, together with the fact that these moments converge. Since all right limits of $J$ have constant first and second moments, it follows from Theorem 4.1 that they all belong to Simon Class. The rest follows from combining (4.3), (4.4), (4.5) and (4.6) together with the definition of $\mathcal{S}(A, B)$. 
Corollary 4.2 is closely related to Theorem 2 in [35] - both our assumptions and conclusions are weaker. We also note that our proof is not that much different from the corresponding parts in Simon's proof. However, we believe that the "right limit point of view" makes various ideas especially transparent and clear. In particular, we would like to emphasize the following subtle point. While convergence of the first and second moments does not imply weak convergence, by Corollary 4.2 it does imply a certain weak form of weak convergence: it holds along any subsequence on which $J$ has a right limit.

Also, it is now clear that any additional condition forcing weak convergence of $\mu_{n}$ is equivalent to a condition that distinguishes a particular element of $\mathcal{S}\left(\tilde{A}-\tilde{B}^{2}, \tilde{B}\right)$ (up to a shift). Computing powers of $J$ shows that the third moment is not enough, but the fourth moment is. Thus we get

Theorem 4.3 (Simon [35]). If (4.5) and (4.6) hold and $\lim _{n \rightarrow \infty} \int x^{4} d \mu_{n}(x)$ exists, then $J$ is bounded, $d \mu_{n}$ converge weakly and $J$ has a unique right limit (up to a shift) in $\mathcal{S}\left(\tilde{A}-\tilde{B}^{2}, \tilde{B}\right)$.

We next want to demonstrate how ratio asymptotics also fit naturally into this framework.

Definition 4.4. Let $\mu$ be a probability measure on the real line. We say $\mu$ is ratio asymptotic if

$$
\lim _{n \rightarrow \infty} \frac{P_{n+1}(z)}{P_{n}(z)} \equiv \lim _{n \rightarrow \infty} \frac{a_{n+1} p_{n+1}(z)}{p_{n}(z)}
$$

exists for all $z \in \mathbb{C} \backslash \mathbb{R}$, where $P_{n}$ are the monic orthogonal polynomials, $p_{n}$ are the orthonormal polynomials, and $a_{n}$ are the off-diagonal Jacobi parameters corresponding to $\mu$.

As above, our strategy for dealing with ratio asymptotic measures consists of identifying the appropriate class of right limits by analyzing their invariants. We also want to emphasize the relationship with weak asymptotic convergence. Let $H\left(\left\{a_{n}, b_{n}\right\}_{n \in \mathbb{Z}}\right)$ be a whole-line Jacobi matrix. Let $J_{n}^{+}=J\left(\left\{a_{j+n}, b_{j+n}\right\}_{j=1}^{\infty}\right)$ be the half-line Jacobi matrix one gets when restricting $H$ to $\ell^{2}(j>n)$ with Dirichlet boundary conditions, and $J_{n}^{-}=J\left(\left\{a_{n-j}, b_{n+1-j}\right\}_{j=1}^{\infty}\right)$, the half-line Jacobi matrix one gets when restricting $H$ to $\ell^{2}(j \leq n)$ with Dirichlet boundary conditions. $J_{n}^{+}$ and $J_{n}^{-}$have spectral measures associated with them which we denote by $\mu_{n}^{+}$and $\mu_{n}^{-}$. Finally, for $z \in \mathbb{C} \backslash \mathbb{R}$ let $m_{ \pm}(n ; z)=\int \frac{d \mu_{n}^{ \pm}(x)}{x-z}$ be the corresponding BorelStieltjes transforms. We are interested in $H$ for which these are constants in $n$. The reason for this is the fact that if $H$ is a right limit of $J$, then $-\frac{1}{m_{-}(0 ; z)}$ is a limit of $\frac{P_{n+1}(z)}{P_{n}(z)}$ along an appropriate subsequence (see e.g. [33]-note that his $m_{-}$ is our $\left.-1 / m_{-}\right)$. Thus,

Theorem 4.5. Let $H\left(\left\{a_{n}, b_{n}\right\}_{n \in \mathbb{Z}}\right)$ be a whole-line Jacobi matrix. Then the following are equivalent:

(i) H belongs to Simon Class and its spectrum is a single interval.

(ii) $a_{n}=a, b_{n}=b$ for some numbers, $a \geq 0$ and $b \in \mathbb{R}$ and all $n \in \mathbb{Z}$.

(iii) $m_{-}(n ; z)=m_{-}(n+1 ; z)$ for all $z \in \mathbb{C} \backslash \mathbb{R}, n \in \mathbb{Z}$.

(iv) $m_{+}(n ; z)=m_{+}(n+1 ; z)$ for all $z \in \mathbb{C} \backslash \mathbb{R}, n \in \mathbb{Z}$.

(v) $m_{-}(n ; z)=m_{-}(n+1 ; z)$ for some $z \in \mathbb{C} \backslash \mathbb{R}$ and all $n \in \mathbb{Z}$.

(vi) $m_{+}(n ; z)=m_{+}(n+1 ; z)$ for some $z \in \mathbb{C} \backslash \mathbb{R}$ and all $n \in \mathbb{Z}$. 
Proof. $(i) \Leftrightarrow($ ii $)$ follows from the theory of periodic Jacobi matrices (see [43, Sect. 7.4]). (ii) $\Rightarrow(i i i) \Rightarrow(v)$ and $(i i) \Rightarrow(i v) \Rightarrow(v i)$ are clear by periodicity. Thus we are left with showing $(v) \Rightarrow(i i)$ and $(v i) \Rightarrow(i i)$. Writing down the continued fraction expansion for $m_{-}(n ; z)$ :

$$
-\frac{1}{m_{-}(n+1 ; z)}=z-b_{n+1}+a_{n}^{2} m_{-}(n ; z), \quad n \in \mathbb{Z},
$$

one sees that $(v)$ implies $m_{-}(n ; z)$ satisfies a quadratic equation. $a_{n}$ and $b_{n+1}$ are then determined from this equation by taking imaginary and real parts, and so we get $(v) \Rightarrow(i i)$ (see the proof of Theorem 2.2 in [35] for details). The same can be done for $m_{+}(n ; z)$ to get $(v i) \Rightarrow(i i)$.

By the above discussion and Theorem 4.5 , it follows that $\mu$ is ratio asymptotic if and only if its Jacobi matrix has a unique right limit in Simon Class with constant off-diagonal elements. Moreover, $(v)$ in Theorem 4.5 implies that it is enough to require ratio asymptotics at a single $z \in \mathbb{C} \backslash \mathbb{R}$. This is precisely the content of Theorem 1 in [35]. We shall show below that the same strategy can be applied in the OPUC case in order to get a strengthening of corresponding results by Khrushchev.

4.2. The Khrushchev Class. We now turn to the discussion of the analogous theory for half-line CMV matrices. Namely, we study CMV matrices with the property that $d \mu_{n}(\theta)=\left|\varphi_{n}\left(e^{i \theta}\right)\right|^{2} d \mu(\theta)$ has a weak limit as $n \rightarrow \infty$. Again, as is clear from the discussion in the introduction, all these right limits belong to Khrushchev Class (recall Definition 1.10) and so the analysis is mainly the analysis of properties of that class. Since nontrivial CMV matrices can have many powers with zero diagonal, the computations are substantially more complicated. Here is the analog of Theorem 4.1:

Theorem 4.6. Let $\mathcal{E}$ be a whole-line $C M V$ matrix and $k \in \mathbb{N} \cup\{\infty\}$. Then the following are equivalent:

(i) $\mathcal{E}$ belongs to Khrushchev Class with $\left[\mathcal{E}^{\ell}\right]_{n, n}=0$ for $\ell=1, \ldots, k-1$ and all $n \in \mathbb{Z}$, and in the case $k<\infty,\left[\mathcal{E}^{k}\right]_{n, n}=c$ for some $c \in \overline{\mathbb{D}} \backslash\{0\}$ and all $n \in \mathbb{Z}$.

(ii) For $\ell=1, \ldots, k-1$,

$$
\int_{0}^{2 \pi} e^{i \ell \theta} d \mu_{n}(\theta)=0, \quad n \in \mathbb{Z}
$$

and if $k<\infty$ then additionally, for some $c \in \overline{\mathbb{D}} \backslash\{0\}$,

$$
\int_{0}^{2 \pi} e^{i k \theta} d \mu_{n}(\theta)=c, \quad n \in \mathbb{Z}
$$

(iii) There exist $n_{0} \in \mathbb{N}, a, b \in(0,1]$, and $t \in[0,2 \pi)$ such that in the case $k<\infty$,

$$
\begin{aligned}
& \left|\alpha_{n_{0}+2 n k}\right|=a, \quad\left|\alpha_{n_{0}+(2 n+1) k}\right|=b, \\
& \alpha_{n_{0}+n k+j}=0, \quad \arg \left(\bar{\alpha}_{n_{0}+(n+1) k} \alpha_{n_{0}+n k}\right)=t, \quad n \in \mathbb{Z}, j=1, \ldots, k-1,
\end{aligned}
$$

and in the case $k=\infty$,

$$
\alpha_{j}=0, \quad j \in \mathbb{Z} \backslash\left\{n_{0}\right\} .
$$

Remark. In particular, this shows that the constancy of the first $k$ moments, where the $k$-th moment is the first nonzero one, implies that $\mathcal{E}$ belongs to Khrushchev Class. Note, however, that the value of the $k$-th moment does not determine the element of the class itself (again, not even up to translation; see Theorem 4.8 
below). Thus, it makes sense to define $\mathcal{K}(c, k)$, for $k<\infty$, to be the set of all matrices in the Khrushchev Class with $\left[\mathcal{E}^{\ell}\right]_{n, n}=0$ for all $n \in \mathbb{Z}, \ell=1, \ldots, k-1$, and $\left[\mathcal{E}^{k}\right]_{n, n}=c \neq 0$ for all $n \in \mathbb{Z}$. In the case $k=\infty$, let $\mathcal{K}(\infty)$ be the set of all matrices with $\left[\mathcal{E}^{\ell}\right]_{n, n}=0$ for all $n \in \mathbb{Z}, \ell \geq 1$. We note that every CMV matrix $\mathcal{E}$ from the Khrushchev Class belongs to one of $\mathcal{K}(c, k), c \in \overline{\mathbb{D}} \backslash\{0\}, k \in \mathbb{N}$, or to $\mathcal{K}(\infty)$.

Proof. $(i) \Rightarrow(i i)$ : Follows from

$$
\int_{0}^{2 \pi} e^{i \ell \theta} d \mu_{n}(\theta)=\left[\mathcal{E}^{\ell}\right]_{n, n} \text { for all } \ell \in \mathbb{N}, n \in \mathbb{Z} .
$$

(ii) $\Rightarrow$ (iii): First, observe that (iii) is equivalent to the following López-type condition: there exists $n_{0} \in \mathbb{Z}$ such that for all $n \in \mathbb{Z}, \ell=1, \ldots, k, j=0, \ldots, \ell-1$,

$$
\bar{\alpha}_{n_{0}+n \ell+j} \alpha_{n_{0}+(n-1) \ell+j}= \begin{cases}a b e^{i t} & j=0, \ell=k, \\ 0 & \text { otherwise. }\end{cases}
$$

We will show that (4.8) holds with $a b e^{i t}=-c$ by verifying inductively with respect to $\ell$ that

$$
-\bar{\alpha}_{n_{0}+n \ell+j} \alpha_{n_{0}+(n-1) \ell+j}= \begin{cases}\int_{0}^{2 \pi} e^{i \ell \theta} d \mu_{n_{0}+n \ell} & j=0, \\ 0 & \text { otherwise }\end{cases}
$$

for some $n_{0} \in \mathbb{Z}$ and all $n \in \mathbb{Z}, \ell=1, \ldots, k, j=0, \ldots, \ell-1$.

The case $\ell=1$ trivially follows from (3.1) since the first moment of $\mu_{n}$ is exactly the diagonal element $\mathcal{E}_{n, n}$ for all $n \in \mathbb{Z}$.

Now suppose (4.9) holds for $\ell=1, \ldots, p-1$ for some $p \leq k$. In view of (4.7), we want to compute $\left[\mathcal{E}^{p}\right]_{n, n}=\left[(\mathcal{L M})^{p}\right]_{n, n}$. To do this, it turns out to be useful to separate the diagonal and off-diagonal elements of $\mathcal{L}$ and $\mathcal{M}$ and identify the contributions to the product. Thus, let the diagonal matrices $X_{-1}=\operatorname{diag} \mathcal{L}$ and $X_{1}=\operatorname{diag} \mathcal{M}$ be the diagonals of $\mathcal{L}$ and $\mathcal{M}$ respectively. Furthermore, define $Y_{-1}$ and $Y_{1}$ through $\mathcal{L}=X_{-1}+Y_{-1}, \mathcal{M}=X_{1}+Y_{1}$. Expressed in this notation, our objective is to compute the diagonal elements of

$$
\mathcal{E}^{p}=\left(X_{-1}+Y_{-1}\right)\left(X_{(-1)^{2}}+Y_{(-1)^{2}}\right) \cdots\left(X_{(-1)^{2 p}}+Y_{(-1)^{2 p}}\right) .
$$

First, it is a direct computation to verify that for any two $s, r \in \mathbb{N}$,

$$
\operatorname{diag} Y_{(-1)^{s}} Y_{(-1)^{s+1}} \cdots Y_{(-1)^{s+r}}=0 .
$$

Now, using (ii) and the induction hypothesis ((4.9) for $\ell \leq p-1)$ one verifies that

$$
\begin{aligned}
& {\left[Y_{(-1)^{j-s}} Y_{(-1)^{j-s+1}} \cdots Y_{(-1)^{j-1}} X_{(-1)^{j}} Y_{(-1)^{j+1}} \cdots Y_{(-1)^{j+s-1}} Y_{(-1)^{j+s}}\right]_{n, n}} \\
& \quad= \begin{cases}\bar{\alpha}_{n+s} \rho_{n}^{2} \cdots \rho_{n+s-1}^{2} & n+s+j \text { is odd }, \\
-\alpha_{n-s-1} \rho_{n-s}^{2} \cdots \rho_{n-1}^{2} & n+s+j \text { is even }\end{cases} \\
& \quad=\left\{\begin{array}{ll}
\bar{\alpha}_{n+s} & n+s+j \text { is odd, } \\
-\alpha_{n-s-1} & n+s+j \text { is even, }
\end{array} \quad n, j \in \mathbb{Z}, s=0, \ldots, p-1,\right.
\end{aligned}
$$

and

$$
\left[Y_{(-1)^{j-s}} Y_{(-1)^{j-s+1}} \cdots Y_{(-1)^{j-1}} X_{(-1)^{j}} Y_{(-1)^{j+1}} \cdots Y_{(-1)^{j+s-1}} Y_{(-1)^{j+s}}\right]_{n, m}=0
$$

whenever $n \neq m$. 
This identity combined with the induction hypothesis, (ii), (4.7), (4.10), and (4.11) implies that (for notational simplicity we let $\widetilde{Y}_{j}=Y_{(-1)^{j}}, \widetilde{X}_{j}=X_{(-1)^{j}}$ )

$$
\begin{aligned}
\int_{0}^{2 \pi} & e^{i p \theta} d \mu_{n}(\theta)=\left[\mathcal{E}^{p}\right]_{n, n} \\
= & \sum_{\ell=1}^{p}\left[\widetilde{Y}_{1} \cdots \widetilde{Y}_{\ell-1} \widetilde{X}_{\ell} \widetilde{Y}_{\ell+1} \cdots \widetilde{Y}_{p+\ell-1} \widetilde{X}_{p+\ell} \widetilde{Y}_{p+\ell+1} \cdots \widetilde{Y}_{2 p}\right]_{n, n} \\
= & -\sum_{\ell=1}^{p} \begin{cases}\bar{\alpha}_{n+p-\ell} \alpha_{n-\ell} & n \text { is odd }, \\
\bar{\alpha}_{n+\ell-1} \alpha_{n-p+\ell-1} & n \text { is even }\end{cases} \\
= & -\sum_{\ell=1}^{p} \bar{\alpha}_{n+p-\ell} \alpha_{n-\ell}, \quad n \in \mathbb{Z} .
\end{aligned}
$$

The idea behind the computation is that all summands containing no $X$, a single $X$, or two $X$ 's that are a distance greater than or less than $p$ apart do not contribute to the diagonal. This follows from the induction hypothesis and (4.11)-(4.13).

Now, observe that the sum in the above equality may have at most one nonzero term. Indeed, if there are no nonzero terms we are done (this may happen only if $p<k$ ), otherwise let $n_{0} \in \mathbb{Z}$ be such that $\bar{\alpha}_{n_{0}} \alpha_{n_{0}-p} \neq 0$. Then combining the induction hypothesis (4.9) with (ii) yields,

$$
\bar{\alpha}_{n+\ell} \alpha_{n}=0, \quad n \in \mathbb{Z}, \ell=1, \ldots, p-1
$$

which together with $\bar{\alpha}_{n_{0}} \alpha_{n_{0}-p} \neq 0$ implies

$$
\alpha_{n_{0}+n p+\ell}=0, \quad n \in \mathbb{Z}, \ell=1, \ldots, p-1 .
$$

Hence, when $p<k$,

$$
0=\int_{0}^{2 \pi} e^{i p \theta} d \mu_{n_{0}+n p}(\theta)=-\bar{\alpha}_{n_{0}+n p} \alpha_{n_{0}+(n-1) p}, \quad n \in \mathbb{Z},
$$

and carrying the induction up to $k$,

$$
c=\int_{0}^{2 \pi} e^{i k \theta} d \mu_{n_{0}+n k}(\theta)=-\bar{\alpha}_{n_{0}+n k} \alpha_{n_{0}+(n-1) k}, \quad n \in \mathbb{Z} .
$$

Thus, (4.9) holds for $\ell=k$, and hence (iii) follows from (4.8) with abe $e^{i t}=-c$.

$($ iii $) \Rightarrow(i)$ : First, note that if $k=\infty$ then there is at most one nonzero Verblunsky coefficient and hence by Example 1.7, $\mathcal{E}$ is in the Khrushchev Class with $d \mu_{n}(\theta)=\frac{d \theta}{2 \pi}$ for all $n \in \mathbb{Z}$, and hence it follows from (4.7) that $\left[\mathcal{E}^{\ell}\right]_{n, n}=0$ for all $\ell \in \mathbb{N}$ and $n \in \mathbb{Z}$.

Next suppose $k<\infty$. It follows from (iii) that there are $t_{0}, t \in[0,2 \pi)$ such that

$$
\alpha_{n_{0}+n}=\left|\alpha_{n_{0}+n}\right| e^{i\left(t_{0}+t n\right)} \text { for all } n \in \mathbb{Z} .
$$

Then, using the Schur algorithm, one finds the following relations between the functions $f_{ \pm}$associated with $\alpha=\left\{\alpha_{n}\right\}_{n \in \mathbb{Z}}$ and $|\alpha|=\left\{\left|\alpha_{n}\right|\right\}_{n \in \mathbb{Z}}$, respectively,

$$
\begin{aligned}
& f_{+}\left(z, n_{0}+n ; \alpha\right)=e^{i\left(t_{0}+t n\right)} f_{+}\left(e^{-i t} z, n_{0}+n ;|\alpha|\right), \\
& f_{-}\left(z, n_{0}+n ; \alpha\right)=e^{-i\left(t_{0}+t(n-1)\right)} f_{-}\left(e^{-i t} z, n_{0}+n ;|\alpha|\right), \quad n \in \mathbb{Z}, z \in \mathbb{D} .
\end{aligned}
$$


Hence by (1.9) the diagonal Schur functions associated with $\alpha$ and $|\alpha|$ are related by

$$
f\left(z, n_{0}+n ; \alpha\right)=e^{i t} f\left(e^{-i t} z, n_{0}+n ;|\alpha|\right), \quad n \in \mathbb{Z}, z \in \mathbb{D} .
$$

Now, the conditions in (iii) imply,

$$
\begin{aligned}
& f_{+}\left(z, n_{0}+n k+j ;|\alpha|\right)=z^{k-j} f_{+}\left(z, n_{0}+(n+1) k ;|\alpha|\right), \\
& f_{-}\left(z, n_{0}+n k+j ;|\alpha|\right)=z^{j-1} f_{-}\left(z, n_{0}+n k+1 ;|\alpha|\right), \\
& f_{+}\left(z, n_{0}+n k ;|\alpha|\right)=f_{+}\left(z, n_{0}+(n \bmod 2) k ;|\alpha|\right), \\
& f_{-}\left(z, n_{0}+n k+1 ;|\alpha|\right)=-f_{+}\left(z, n_{0}+n k ;|\alpha|\right), \quad n \in \mathbb{Z}, j=1, \ldots, k, z \in \mathbb{D} .
\end{aligned}
$$

These identities together with (1.9) and (4.14) yield

$$
\begin{aligned}
& f\left(z, n_{0}+n k+j ; \alpha\right) \\
& \quad=e^{-i t(k-2)} z^{k-1} f_{+}\left(e^{-i t} z, n_{0}+(n+1) k ;|\alpha|\right) f_{-}\left(e^{-i t} z, n_{0}+n k+1 ;|\alpha|\right) \\
& \quad=-e^{-i t(k-2)} z^{k-1} f_{+}\left(e^{-i t} z, n_{0}+(n+1) k ;|\alpha|\right) f_{+}\left(e^{-i t} z, n_{0}+n k ;|\alpha|\right) \\
& \quad=-e^{-i t(k-2)} z^{k-1} f_{+}\left(e^{-i t} z, n_{0}+k ;|\alpha|\right) f_{+}\left(e^{-i t} z, n_{0} ;|\alpha|\right)
\end{aligned}
$$

for all $n \in \mathbb{Z}, j=1, \ldots, k, z \in \mathbb{D}$. Hence $f(\cdot, n ; \alpha)=f(\cdot, m ; \alpha)$ which is equivalent to $\mu_{m}=\mu_{n}$ for all $m, n \in \mathbb{Z}$.

The presence of the factor $z^{k-1}$ implies that the first $k-1$ moments of $\mu_{n}$, $n \in \mathbb{Z}$, are zero. This follows from the relationship (1.5) between Schur functions and Carathéodory functions and the fact that Taylor coefficients of $F$ are twice the complex conjugates of the moments of $\mu$. Moreover, since $z^{-k+1} f\left(z, n_{0}+n k+j ; \alpha\right)$ is nonzero at the origin, the $k$-th moment of $\mu_{n}, n \in \mathbb{Z}$, is nonzero, and hence one gets $(i)$ from (4.7).

Corollary 4.7. Let $\mathcal{C}$ be a half-line $C M V$ matrix and let $\mu$ be its spectral measure. For $n \geq 0$, let $d \mu_{n}(\theta)=\left|\varphi_{n}\left(e^{i \theta}\right)\right|^{2} d \mu(\theta)$ be the spectral measure of $C$ and $\delta_{n}$. If for some $c \in \overline{\mathbb{D}} \backslash\{0\}$ and $k \in \mathbb{N} \cup\{\infty\}$,

$$
\lim _{n \rightarrow \infty} \int_{0}^{2 \pi} e^{i \ell \theta} d \mu_{n}(\theta)= \begin{cases}0 & \ell=1, \ldots, k-1, \\ c & \ell=k, k<\infty\end{cases}
$$

then all right limits of $\mathcal{C}$ are in $\mathcal{K}(c, k)$ if $k<\infty$ or in $\mathcal{K}(\infty)$ if $k=\infty$.

The analogy with Corollary 4.2 should be clear. Corollary 4.7 is a variant of Theorem E in [19] with weaker assumptions and weaker conclusions. Our proof is new and based on a completely different approach. We also note that much the same as in the Jacobi case, convergence of the first $k$-moments does not imply weak convergence, but by Corollary 4.7 it does imply the same weak form of weak convergence: convergence holds along any subsequence on which $\mathcal{C}$ has a right limit.

A notable difference between the OPUC and OPRL cases is the fact that multiplication of the Verblunsky coefficients by a constant phase does not change the spectral measures. Thus, even when $\mu_{n}$ converges weakly, it is not possible to deduce uniqueness of a right limit (even up to a shift). Note that the phase ambiguity is equivalent to a choice of $t_{0}$ in the proof of Theorem 4.6 and there is no way to determine this $t_{0}$ from information on $\mu_{n}$ alone. In the case $k=\infty$ even $\left|\alpha_{n_{0}}\right|$ cannot be determined from the information on the measure and so the indeterminacy is, in a sense, even more severe. 
That said, as in the Jacobi case, it is clear that when $k<\infty$ any condition forcing weak convergence of $\mu_{n}$ (in addition to those in Corollary 4.7) is equivalent to a condition that distinguishes an element of $\mathcal{K}(c, k)$ (up to a shift and multiplication by an arbitrary phase). In particular, a somewhat tedious computation (along the lines of the argument in $(i i) \Rightarrow($ iii $)$ above) shows that the following result holds:

Theorem 4.8. Suppose that $k<\infty$, (4.15) holds, and $\lim _{n \rightarrow \infty} \int_{0}^{2 \pi} e^{2 i k \theta} d \mu_{n}(\theta)$ exists. Then $d \mu_{n}$ converge weakly and $\mathcal{C}$ has a unique right limit (up to a shift and multiplication by a constant phase) in $\mathcal{K}(c, k)$.

Remark. We note that on the level of Verblunsky coefficients, Theorems 4.6 and 4.8 imply for $k<\infty$,

$$
\begin{aligned}
& \lim _{n \rightarrow \infty}\left|\alpha_{n_{0}+2 n k+j}\right|= \begin{cases}a & j=0, \\
b & j=k, \\
0 & j \in\{1, \ldots, k-1, k+1, \ldots, 2 k-1\},\end{cases} \\
& \lim _{n \rightarrow \infty} \bar{\alpha}_{n_{0}+(n+1) k} \alpha_{n_{0}+n k}=-c, \text { for some } n_{0} \in \mathbb{Z} \text { and } a b=|c|,
\end{aligned}
$$

and similarly, Theorem 4.6 and Corollary 4.7 imply for $k=\infty$,

$$
\lim _{n \rightarrow \infty}\left|\alpha_{n_{0}+n} \alpha_{n}\right|=0 \text { for any } n_{0} \in \mathbb{Z} \text {. }
$$

This extends [19, Thm. E], where the stronger condition of weak convergence for the measures $d \mu_{n}$ is assumed.

Next, we use right limits to study ratio asymptotics. It is convenient to introduce $\widetilde{\mathcal{K}}(c, 1)$ as the subclass of $\mathcal{K}(c, 1)$ consisting of CMV matrices with Verblunsky coefficients of constant absolute value.

Definition 4.9. Let $\mu$ be a probability measure on the unit circle. We say $\mu$ is ratio asymptotic if

$$
\lim _{n \rightarrow \infty} \frac{\Phi_{n+1}^{*}(z)}{\Phi_{n}^{*}(z)}
$$

exists for all $z \in \mathbb{D}$, where, as usual, $\Phi_{n}(z)$ is the degree $n$ monic orthogonal polynomial associated to $\mu$.

In particular, we say ratio asymptotics holds at $z \in \mathbb{D}$ with limit $G(z)$ if

$$
\lim _{n \rightarrow \infty} \frac{\Phi_{n+1}^{*}(z)}{\Phi_{n}^{*}(z)}=G(z) .
$$

Theorem 4.10. Let $\Phi_{n}$ be the monic orthogonal polynomials associated with a half-line $C M V$ matrix $\mathcal{C}$. If either all right limits of $\mathcal{C}$ are in $\mathcal{K}(\infty)$ or $\mathcal{C}$ has a unique right limit (up to a multiplication by a constant phase) in $\widetilde{\mathcal{K}}(c, 1)$, then $\mu$ is ratio asymptotic.

Conversely, if ratio asymptotics holds at some point $z_{0} \in \mathbb{D} \backslash\{0\}$ with limit $G\left(z_{0}\right)=1$, then all right limits of $\mathcal{C}$ are in $\mathcal{K}(\infty)$. If ratio asymptotics holds at two points $z_{1}, z_{2} \in \mathbb{D} \backslash\{0\}$ and the limit is not 1 at either point, then $\mathcal{C}$ has a unique right limit (up to multiplication by a constant phase) in $\widetilde{\mathcal{K}}(c, 1)$ for some $c \in \overline{\mathbb{D}} \backslash\{0\}$.

Proof. First, observe that it follows from the Szegö recursion (1.1) that for all $n \in \mathbb{Z}_{+}$and $z \in \mathbb{D}$,

$$
1-\frac{\Phi_{n+1}^{*}(z)}{\Phi_{n}^{*}(z)}=z \alpha_{n} \frac{\Phi_{n}(z)}{\Phi_{n}^{*}(z)}=z \alpha_{n} f\left(z ;-\bar{\alpha}_{n-1},-\bar{\alpha}_{n-1}, \ldots,-\bar{\alpha}_{0}, 1\right) .
$$


We refer to [37, Prop. 9.2.3] for the details on the second equality. Abbreviating by $f_{n}(z)=f\left(z ;-\bar{\alpha}_{n-1},-\bar{\alpha}_{n-1}, \ldots,-\bar{\alpha}_{0}, 1\right)$, we see that ratio asymptotics (4.18) at $z \in \mathbb{D} \backslash\{0\}$ is equivalent to $\lim _{n \rightarrow \infty} \alpha_{n} f_{n}(z)=g(z) \equiv(1-G(z)) / z$.

Let $\mathcal{E}$ be a right limit of $\mathcal{C}$ and $\beta_{n}, f_{ \pm}(\cdot, n), n \in \mathbb{Z}$, be the Verblunsky coefficients and Schur functions associated with $\mathcal{E}$. Then, $\beta_{n} f_{-}(z, n)=\lim _{j \rightarrow \infty} \alpha_{n+n_{j}} f_{n+n_{j}}(z)$ for all $n \in \mathbb{Z}, z \in \mathbb{D}$, and some sequence $\left\{n_{j}\right\}_{j \in \mathbb{N}}$.

By Theorem 4.6, if $\mathcal{E} \in \mathcal{K}(\infty)$ then at most one $\beta_{n}$ is nonzero, and hence $\beta_{0} f_{-}(z, 0)=0$ for all $z \in \mathbb{D}$. If $\mathcal{E} \in \widetilde{\mathcal{K}}(c, 1)$ then $\left|\beta_{n}\right|=\sqrt{|c|}$ and $\bar{\beta}_{n+1} \beta_{n}=-c$ for all $n \in \mathbb{Z}$. Then the Schur algorithm implies that $\beta_{0} f_{-}(z, 0)$ is a function that depends only on the value of $c$. Since in both cases $\beta_{0} f_{-}(z, 0)$ is independent of the sequence $n_{j}$, it follows that $\lim _{n \rightarrow \infty} \alpha_{n} f_{n}(z)=\beta_{0} f_{-}(z, 0)$ for all $z \in \mathbb{D}$. Thus, by (4.19), ratio asymptotics holds for all $z \in \mathbb{D}$.

Conversely, by (4.19), ratio asymptotics at $z \in \mathbb{D} \backslash\{0\}$ implies $\beta_{n} f_{-}(z, n)=g(z)$ for all $n \in \mathbb{Z}$. By the Schur algorithm we have

$$
f_{-}(z, n+1)[1-z g(z)]=z f_{-}(z, n)-\bar{\beta}_{n} \text { for all } n \in \mathbb{Z} .
$$

If (4.18) holds at $z_{0} \neq 0$ and the limit is 1 , then by (4.19) $g\left(z_{0}\right)=0$. Thus, by (4.20) there is at most one nonzero $\beta_{n}$ since $\beta_{n_{0}} \neq 0$ implies inductively that $f_{-}\left(z_{0}, n\right) \neq 0$ and hence $\beta_{n}=0$ (since $g\left(z_{0}\right)=0$ ) for all $n>n_{0}$. By Theorem 4.6, $\mathcal{E} \in \mathcal{K}(\infty)$.

Finally consider the case where ratio asymptotics holds at two different points $z_{1}, z_{2} \in \mathbb{D} \backslash\{0\}$ and the limit is not 1 at either point. Then by (4.19), $g\left(z_{1}\right) \neq 0$ and $g\left(z_{2}\right) \neq 0$ and hence $\beta_{n} \neq 0$ for all $n \in \mathbb{Z}$. We also see that $z_{1} g\left(z_{1}\right) \neq z_{2} g\left(z_{2}\right)$ since otherwise it follows from (4.20) that $z_{1}=z_{2}$. Thus, multiplying (4.20) by $\beta_{n+1} /(z g(z))$, substituting $z=z_{j}, j=1,2$, and subtracting the results then yields

$$
\beta_{n+1} \bar{\beta}_{n}=\frac{\frac{1}{z_{1}}-g\left(z_{1}\right)-\frac{1}{z_{2}}+g\left(z_{2}\right)}{\frac{1}{z_{1} g\left(z_{1}\right)}-\frac{1}{z_{1} g\left(z_{1}\right)}} \text { for all } n \in \mathbb{Z} \text {. }
$$

Similarly, multiplying (4.20) by $\left|\beta_{n}\right|^{2} \beta_{n+1}$ and evaluating at $z=z_{1}$ one obtains,

$$
\left|\beta_{n}\right|^{2}=\frac{z_{1} g\left(z_{1}\right) \beta_{n+1} \bar{\beta}_{n}}{g\left(z_{1}\right)\left(1-z_{1} g\left(z_{1}\right)\right)+\beta_{n+1} \bar{\beta}_{n}} \text { for all } n \in \mathbb{Z} .
$$

By (4.21) the right-hand side of (4.22) is $n$-independent, and hence $\left|\beta_{n}\right|$ as well as $\beta_{n+1} \bar{\beta}_{n}$ are $n$-independent constants uniquely determined by the ratio asymptotics (4.18) at $z_{1}$ and $z_{2}$. Thus, $\mathcal{E} \in \widetilde{\mathcal{K}}(c, 1)$ with $c=-\bar{\beta}_{n+1} \beta_{n} \neq 0$. Since $c$ is determined by the ratio asymptotics at $z_{1}$ and $z_{2}$, all right limits are the same up to multiplication by a constant phase.

Remark. This theorem extends an earlier result of Khrushchev [19, Thm. A].

We conclude with the

Proof of Theorem 1.13. Let $H$ belong to the Simon Class and let $a, b, c$ be as in (iii) of Theorem 4.1. If $a, c>0$ then $H$ is a periodic whole-line Jacobi matrix which is well known to be reflectionless on its spectrum ([43]). If $a=0$ or $c=0$ then $H$ is a direct sum of identical $2 \times 2$ (or $1 \times 1$ ) self-adjoint matrices and so has pure point spectrum of infinite multiplicity supported on at most two points.

For $\mathcal{E}$ in the Khrushchev Class with $k<\infty$ the same analysis goes through: as long as $|a|,|b|<1$ we get a reflectionless operator. If one of them or both are unimodular then it is easy to see that $\mathcal{E}$ is a direct sum of $2 \times 2$ (or $1 \times 1)$ matrices. 
If $k=\infty$ then $\mathcal{E}$ is either reflectionless or belongs to the class of matrices from Example 1.7.

\section{REFERENCES}

[1] S. V. Breimesser and D. B. Pearson, Asymptotic value distribution for solutions of the Schrödinger equation, Math. Phys. Anal. Geom. 3 (2000), 385-403.

[2] S. V. Breimesser and D. B. Pearson, Geometrical aspects of spectral theory and value distribution for Herglotz functions, Math. Phys. Anal. Geom. 6 (2003), 29-57.

[3] J. Breuer, E. Ryckman, and B. Simon, in preparation.

[4] M. J. Cantero, L. Moral, and L. Velázquez, Five-diagonal matrices and zeros of orthogonal polynomials on the unit circle, Lin. Algebra Appl. 362 (2003), 29-56.

[5] C. De Concini and R. A. Johnson, The algebraic-geometric AKNS potentials, Ergod. Th. Dyn. Syst. 7 (1987), 1-24.

[6] W. Craig, The trace formula for Schrödinger operators on the line, Commun. Math. Phys. 126 (1989), 379-407.

[7] P. Deift and B. Simon, Almost periodic Schrödinger operators III. The absolutely continuous spectrum in one dimension, Commun. Math. Phys. 90 (1983), 389-411.

[8] Ya. L. Geronimus, On polynomials orthogonal on the circle, on trigonometric moment problem, and on allied Carathéodory and Schur functions, Mat. Sb. 15 (1944), 99-130.

[9] F. Gesztesy, M. Krishna, and G. Teschl, On isospectral sets of Jacobi operators, Commun. Math. Phys. 181 (1996), 631-645.

[10] F. Gesztesy, K. A. Makarov, and M. Zinchenko, Local AC spectrum for reflectionless Jacobi, CMV, and Schrödinger operators, Acta Appl. Math. 103 (2008), 315-339.

[11] F. Gesztesy and P. Yuditskii, Spectral properties of a class of reflectionless Schrödinger operators, J. Funct. Anal. 241 (2006), 486-527.

[12] F. Gesztesy and M. Zinchenko, A Borg-type theorem associated with orthogonal polynomials on the unit circle, J. Lond. Math. Soc. (2) 74 (2006), 757-777.

[13] F. Gesztesy and M. Zinchenko, Weyl-Titchmarsh theory for CMV operators associated with orthogonal polynomials on the unit circle, J. Approx. Theory 139 (2006), 172-213.

[14] F. Gesztesy and M. Zinchenko, Local spectral properties of reflectionless Jacobi, CMV, and Schrödinger operators, J. Diff. Eqs. 246 (2009), 78-107.

[15] L. Golinskii and P. Nevai, Szego difference equations, transfer matrices and orthogonal polynomials on the unit circle, Commun. Math. Phys. 223 (2001), 223-259.

[16] V. Jakšić and Y. Last, Spectral structure of Anderson type Hamiltonians, Invent. Math. 141 (2000), 561-577.

[17] R. A. Johnson, The recurrent Hill's equation, J. Diff. Eqs. 46 (1982), 165-193.

[18] S. Khrushchev, Schur's algorithm, orthogonal polynomials, and convergence of Wall's continued fractions in $L^{2}(\mathbb{T})$, J. Approx. Theory 108 (2001), 161-248.

[19] S. Khrushchev, Classification theorems for general orthogonal polynomials on the unit circle, J. Approx. Theory 116 (2002), 268-342.

[20] S. Kotani, Ljapunov indices determine absolutely continuous spectra of stationary random one-dimensional Schrödinger operators, in "Stochastic Analysis", K. Itǒ (ed.), NorthHolland, Amsterdam, 1984, pp. 225-247.

[21] S. Kotani, One-dimensional random Schrödinger operators and Herglotz functions, in "Probabilistic Methods in Mathematical Physics", K. Itǒ and N. Ikeda (eds.), Academic Press, New York, 1987, pp. 219-250.

[22] S. Kotani and M. Krishna, Almost periodicity of some random potentials, J. Funct. Anal. 78 (1988), 390-405.

[23] Y. Last and B. Simon, The essential spectrum of Schrödinger, Jacobi, and CMV operators, J. Anal. Math. 98 (2006), 183-220.

[24] M. Melnikov, A. Poltoratski, and A. Volberg, Uniqueness theorems for Cauchy integrals, Preprint (2007), arXiv: math-cv/0704.0621

[25] F. Nazarov, A. Volberg, and P. Yuditskii, Reflectionless measures with a point mass and singular continuous component, Preprint (2007), arXiv:math-ph/0711.0948

[26] R. Nevanlinna, Analytic Functions, translated from the second German edition by Phillip Emig, Die Grundlehren der mathematischen Wissenschaften, Band 162, Springer-Verlag, New York-Berlin, 1970. 
[27] F. Peherstorfer and P. Yuditskii, Asymptotic behavior of polynomials orthonormal on a homogeneous set, J. Anal. Math. 89 (2003), 113-154.

[28] F. Peherstorfer and P. Yuditskii, Almost periodic Verblunsky coefficients and reproducing kernels on Riemann surfaces, J. Approx. Theory 139 (2006), 91-106.

[29] A. Poltoratski and C. Remling, Reflectionless Herglotz functions and Jacobi matrices, to appear in Commun. Math. Phys.

[30] E. A. Rakhmanov, On the asymptotics of the ratio of orthogonal polynomials, Math. USSR Sb. 32 (1977), 199-213.

[31] E. A. Rakhmanov, On the asymptotics of the ratio of orthogonal polynomials, II, Math. USSR Sb. 46 (1983), 105-117.

[32] C. Remling, The absolutely continuous spectrum of one-dimensional Schrödinger operators, Math. Phys. Anal. Geom. 10 (2007), 359-373.

[33] C. Remling, The absolutely continuous spectrum of Jacobi matrices, Preprint (2007), arXiv:math-sp/0706.1101

[34] B. Simon The classical moment problem as a self-adjoint finite difference operator, Adv. Math. 137 (1998), 82-203.

[35] B. Simon, Ratio asymptotics and weak asymptotic measures for orthogonal polynomials on the real line, J. Approx. Theory 126 (2004), 198-217.

[36] B. Simon, Orthogonal Polynomials on the Unit Circle, Part 1: Classical Theory, AMS Colloquium Series, 54.1, American Mathematical Society, Providence, RI, 2005.

[37] B. Simon, Orthogonal Polynomials on the Unit Circle, Part 2: Spectral Theory, AMS Colloquium Series, 54.2, American Mathematical Society, Providence, RI, 2005.

[38] R. Sims, Reflectionless Sturm-Liouville equations, J. Comp. Appl. Math. 208 (2007), 207225.

[39] M. Sodin and P. Yuditskii, Almost periodic Sturm-Liouville operators with Cantor homogeneous spectrum and pseudoextendible Weyl functions, Russ. Acad. Sci. Dokl. Math. 50 (1995), 512-515.

[40] M. Sodin and P. Yuditskii, Almost periodic Sturm-Liouville operators with Cantor homogeneous spectrum, Comment. Math. Helvetici 70 (1995), 639-658.

[41] M. Sodin and P. Yuditskii, Almost-periodic Sturm-Liouville operators with homogeneous spectrum, in "Algebraic and Geometric Methods in Mathematical Physics", A. Boutel de Monvel and A. Marchenko (eds.), Kluwer, 1996, pp. 455-462.

[42] M. Sodin and P. Yuditskii, Almost periodic Jacobi matrices with homogeneous spectrum, infinite dimensional Jacobi inversion, and Hardy spaces of character-automorphic functions, J. Geom. Anal. 7 (1997), 387-435.

[43] G. Teschl, Jacobi Operators and Completely Integrable Nonlinear Lattices, Mathematical Surveys and Monographs, 72, American Mathematical Society, Providence, RI, 2000.

Mathematics 253-37, California Institute of Technology, Pasadena CA 91125-0001, USA

E-mail address: jbreuer@caltech.edu

E-mail address: eryckman@caltech.edu

E-mail address: maxim@caltech.edu 